ARTICLE

Received 9 Jun 2016 | Accepted 1 Sep 2016 | Published 13 Oct $2016 \quad$ DOl: 10.1038/ncomms13096 OPEN

\title{
Macrophage-derived extracellular vesicle-packaged WNTs rescue intestinal stem cells and enhance survival after radiation injury
}

Subhrajit Saha ${ }^{1, \star}, \dagger$, Evelyn Aranda ${ }^{2, \star}$, Yoku Hayakawa ${ }^{3}$, Payel Bhanja ${ }^{1, \dagger}$, Safinur Atay ${ }^{4}$, N Patrik Brodin ${ }^{1}$, Jiufeng Li $^{2}$, Samuel Asfaha ${ }^{3}$, Laibin Liu', Yagnesh Tailor ${ }^{3}$, Jinghang Zhang ${ }^{5}$, Andrew K. Godwin ${ }^{4}$, Wolfgang A. Tome ${ }^{1}$, Timothy C. Wang ${ }^{3}$, Chandan Guha ${ }^{1,6, \star \star} \&$ Jeffrey W. Pollard ${ }^{2,7, \star \star}$

WNT/ $\beta$-catenin signalling is crucial for intestinal homoeostasis. The intestinal epithelium and stroma are the major source of WNT ligands but their origin and role in intestinal stem cell (ISC) and epithelial repair remains unknown. Macrophages are a major constituent of the intestinal stroma. Here, we analyse the role of macrophage-derived WNT in intestinal repair in mice by inhibiting their release using a macrophage-restricted ablation of Porcupine, a gene essential for WNT synthesis. Such Porcn-depleted mice have normal intestinal morphology but are hypersensitive to radiation injury in the intestine compared with wild-type (WT) littermates. Porcn-null mice are rescued from radiation lethality by treatment with WT but not Porcn-null bone marrow macrophage-conditioned medium (CM). Depletion of extracellular vesicles (EV) from the macrophage CM removes WNT function and its ability to rescue ISCs from radiation lethality. Therefore macrophage-derived EV-packaged WNTs are essential for regenerative response of intestine against radiation.

\footnotetext{
${ }^{1}$ Department of Radiation Oncology, Albert Einstein College of Medicine \& Montefiore Medical Center, Bronx, New York 10461, USA. ${ }^{2}$ Department of Developmental and Molecular Biology, Albert Einstein College of Medicine, Bronx, New York 10461, USA. ${ }^{3}$ Department of Medicine, Division of Digestive and Liver Diseases, Irving Cancer Research Center, Columbia University, New York, New York 10032, USA. ${ }^{4}$ Department of Pathology and Laboratory Medicine, University of Kansas Medical Center, Kansas City, Kansas 66160, USA. ${ }^{5}$ Department of Microbiology and Immunology, Albert Einstein College of Medicine, Bronx, New York, 10461, USA. ${ }^{6}$ Department of Pathology, Albert Einstein College of Medicine, Bronx, New York 10461, USA. ${ }^{7}$ MRC Centre for Reproductive Health, University of Edinburgh, Edinburgh EH16 4TJ, UK. * These authors contributed equally to this work. ${ }^{\dagger}$ Present addresses: Department of Radiation Oncology University of Kansas Medical Center, Kansas City, Kansas 66160, USA (S.S. and P.B.); Department of Cancer Biology, University of Kansas Medical Center, Kansas City, Kansas 66160, USA (S.S.). ${ }^{\star \star}$ These authors jointly supervised this work. Correspondence and requests for materials should be addressed to J.W.P. (email: Jeff.pollard@ed.ac.uk).
} 
ntestinal epithelial homoeostasis depends on the signalling crosstalk between the crypt intestinal stem cells (ISC) and the surrounding niche, including the intestinal subepithelial myofibroblasts, endothelial cells and macrophages. The cells in the ISC niche provide critical growth factor/signals for ISC regeneration and intestinal homoeostasis ${ }^{1}$. We have recently reported that the radiation-induced gastrointestinal syndrome (RIGS) results from a combination of radiation-induced loss of crypt progenitors and stromal cells along with aberrant signalling in the ISC niches ${ }^{2}$. Transplantation of bone marrow-derived adherent stromal cells accelerates the restitution of the irradiated ISC niche, promotes ISC regeneration and improves survival from RIGS, following whole-body irradiation (WBI) in C57Bl/6 mice. Transplantation of the bone marrow-derived adherent stromal cell population depleted of all CD11b ${ }^{+ \text {ve }}$ myeloid cells failed to rescue mice from lethal radiation injury ${ }^{2}$. However, the specific role of bone marrow-derived mature macrophages that are $\mathrm{CD} 11 \mathrm{~b}^{+\mathrm{ve}}$ against lethal radiation injury in the intestine has not been studied.

Evidence in support of a key role for macrophages in crypt regeneration comes from studies where depletion of host macrophages with liposome-encapsulated clodronate resulted in poor survival after irradiation ${ }^{2}$. Macrophages play an important role in coordinating signals from gut microbes and injured epithelium, and thereby transmit regenerative signals to ISC $^{3,4}$. Our previous studies have shown that TLR-mediated activation of macrophages followed by transplantation could ameliorate intestinal injury ${ }^{5}$. Moreover, in a mouse model of colitis, it has been shown TLR-mediated activation of colonic macrophages is critical for regeneration of colonic progenitors ${ }^{4}$. Nevertheless, while these earlier studies have outlined a role for macrophages in promoting intestinal regeneration, the effect of macrophages on survival and proliferation of ISCs and the nature of the signals that are transmitted has not been elucidated.

$\mathrm{Wnt} / \beta$-catenin signalling plays a major role in ISC self-renewal and proliferation and thereby maintenance of intestinal homoeostasis 6,7 . WNT ligands bind to LRP5/6 and Frizzled co-receptors present on epithelial crypt cells, leading to $\beta$-catenin stabilization and nuclear translocation ${ }^{8}$ where it binds to the nuclear transcription factor TCF4 to drive a gene-expression programme that supports stem cell maintenance, proliferation and differentiation. Activation of $\mathrm{WNT} / \beta$-catenin signalling is also crucial for crypt regeneration following injury. Several reports, have demonstrated that Rspondin 1 (RSPO1), an ISC growth factor and LGR5 receptor agonist, activates $\mathrm{WNT} / \beta$ catenin pathway to repair and regenerate the intestine following chemo-radiation-induced injury ${ }^{9-12}$. Systemic administration of DKK1, a negative regulator $\mathrm{WNT} / \beta$-catenin pathway, impairs the RSPO1-induced intestinal regeneration ${ }^{13}$.

WNTs are evolutionarily conserved, cysteine-rich glycoproteins capable of functioning in both a paracrine and autocrine manner. Epithelial and stromal cells of the small intestine express 19 different Wnt genes ${ }^{7}$. These WNT ligands are involved in various physiological processes including injury repair, innate and adaptive immunity, and intermediary metabolism ${ }^{14-16}$. However, the overall importance of secreted WNTs in proliferation and self-renewal of ISCs remains unknown. Some reports demonstrated that ablation of WNTs in the mouse intestine could not inhibit crypt stem cell proliferation in vivo ${ }^{14,17}$. WNT secretion can be inhibited by deletion of Porcn, an indispensable part of the core WNT ligand secretion machinery ${ }^{18,19}$. PORCN is a trans-membrane endoplasmic reticulum $\mathrm{O}$-acyl transferase ${ }^{20}$ encoded by a single copy gene on the $\mathrm{X}$ chromosome and is involved in palmitoleation of all WNT molecules synthesized in the cell ${ }^{21-23}$. Palmitoleation is an essential process for post-translational modification of WNT that is needed for binding of WNTs with wingless, an integral membrane carrier protein that is essential for the secretion of all known vertebrate WNTs and their binding to Frizzled receptors ${ }^{18,24,25}$. It has been reported that mice deficient in all intestinal epithelial WNTs due to deletion of the gene Porcupine (Porcn) by Villin-Cre have normal ISC proliferation and homoeostasis in vivo ${ }^{1}$. However inhibition of WNT release from both epithelial and stromal sources by pharmacological inhibition of Porcupine showed decrease in epithelial proliferation. Moreover it was noted that global pharmacologic but not epithelial-specific inhibition of Porcn significantly increased the radio-sensitivity of intestine ${ }^{1}$. These observations clearly suggest the importance of stromal cell-derived WNTs in intestinal homoeostasis and regeneration.

Macrophages maybe one of the sources of stromal WNTs in the crypt as in other contexts macrophage-derived WNTs have been reported to have a significant role in tissue repair and regeneration ${ }^{26,27}$. For example macrophage-derived WNT7b was shown to be crucial for epithelial regeneration in response to kidney injury ${ }^{28}$ and for hepatocyte regeneration in the liver ${ }^{29}$. In addition, macrophage-derived WNTs have been shown to affect blood vessel formation by regulating VEGF and angiopoietin signalling in vascular endothelial cells ${ }^{27}$.

In this study to define the role of macrophage-derived WNTs in intestinal repair and regeneration following radiation injury, we used a genetic approach to block WNT release from macrophages by ablating a floxed allele of Porcn with a mononuclear phagocyte restricted cre-recombinase expressed from the colony-stimulating factor-1 receptor (Csf1r) promoter (Csf1r.icre (ref. 30). Using this strategy we found that inhibition of macrophage-derived WNTs exacerbated RIGS, impaired recovery from radiation injury with the loss of Lgr5 ${ }^{+\mathrm{ve}} \mathrm{crypt}$ base columnar cells (CBCs) and decreased survival. Treatment with wild-type (WT) bone marrow macrophage (BMM $\phi$ )conditioned medium (CM) or WNT-deficient Porcupine-null $\mathrm{BMM} \phi \mathrm{CM}$ supplemented with exogenous WNT ligands rescued the crypt epithelium from radiation toxicity. We have also demonstrated that macrophage-derived WNTs are expressed by BMMФ and secreted through extracellular vesicle $(E V)^{31}$ and therefore treatment with purified EVs could rescue crypt cells from radiation toxicity. This radioprotective activity was lost on EV depletion from ВMМФ CM indicating the ligands are secreted in EVs. However, deletion of Porcn in macrophages in nonirradiated mice did not show any obvious phenotype in the intestine indicating that the macrophage-derived WNT activity is specific for repair and regeneration. Taken together, these observations indicate that macrophage-derived EV-packaged WNTs are critical for regenerative response of intestine following injury.

\section{Results}

Porcn deletion in M $\phi$ sensitizes gut to lethal doses of WBI. To assess the role of macrophage-derived WNT in intestinal regeneration, mice carrying a floxed allele of $\operatorname{Porcn}^{21}$ were crossed to Csf1r.iCre male mice ${ }^{30}$ to generate Csf1r.iCre;Porcn fl/fl mice that will have a deletion of Porcn restricted to macrophages. Analysis of BMM $\phi$-derived DNA from the Porcn ${ }^{f l f l}$ mice with or without cre showed efficient ablation of the floxed allele in the presence of Csflr.icre with loss of the $248 \mathrm{bp}$ floxed allele and generation of a $386 \mathrm{bp}$ recombined allele (Supplementary Fig. 1a). Macrophage-specific deletion of Porcn was confirmed at the expression level by PCR of DNA isolated from BMM $\phi$, pulmonary and crypt epithelial cells and hepatocytes of Csf1r.iCre;Porcn fl/fl mice. The presence of porcupine floxed allele was detected in all the tissue samples except in the 
targeted BMM $\phi$ (Supplementary Fig. 1b). Quantitative real-time (QPCR) PCR using both RNA and DNA of the cre-containing versus cre-negative $\mathrm{BMM} \phi$ indicated $\sim 80 \%$ allele ablation typical for floxed alleles (Supplementary Fig. 1c,d respectively). A TCF/LEF (TOPFLASH) reporter assay ${ }^{32}$ was used to measure
WNT/ $\beta$-catenin activity and this assay demonstrated that $\mathrm{CM}$ from Csf1r.iCre;Porcn flfl mice BMM $\phi$ failed to activate the $\beta$-catenin pathway whereas Cre-negative littermates referred to as WT BMM $\phi$ showed activity (Supplementary Fig. 1e). Pharmacological inhibition of Porcupine in macrophages using a
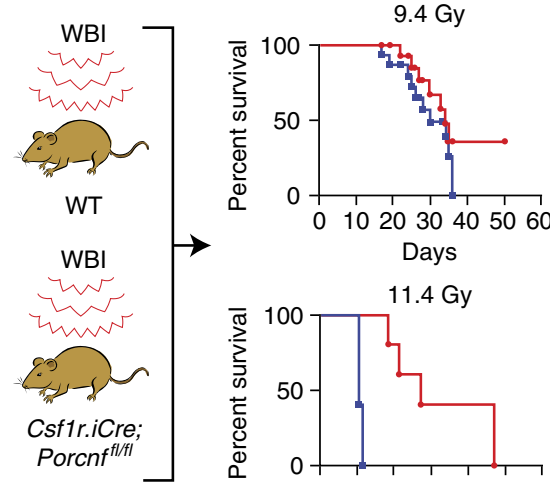

Days

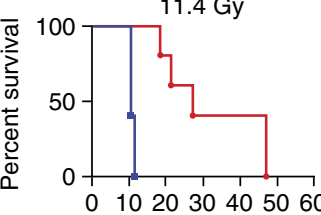

Days

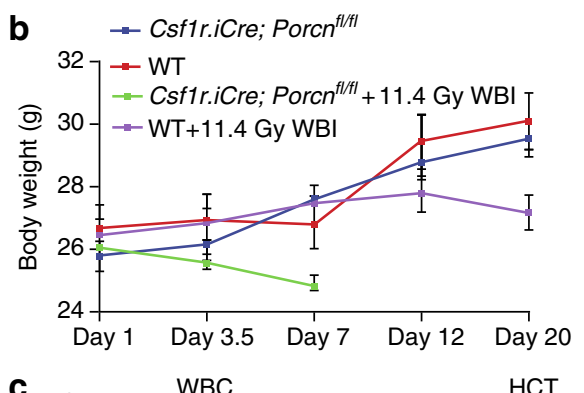

C 4
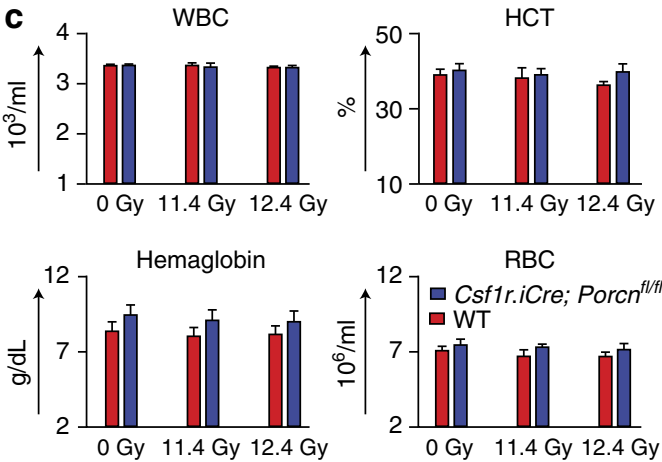

d
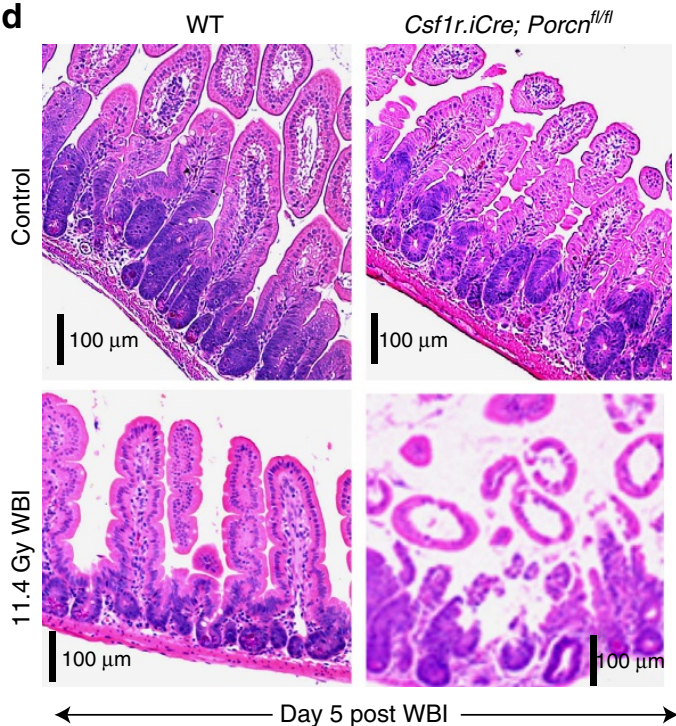
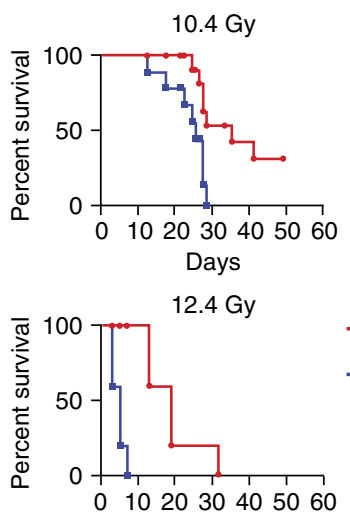

Days

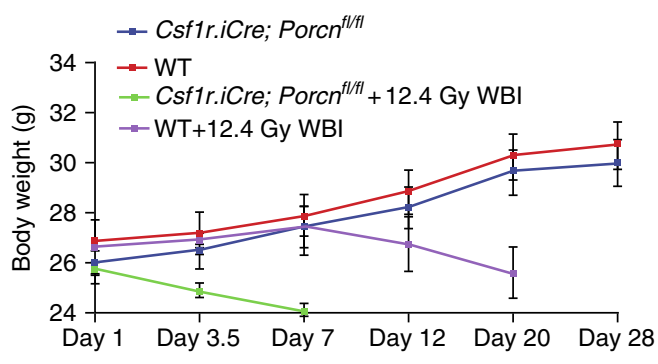

e

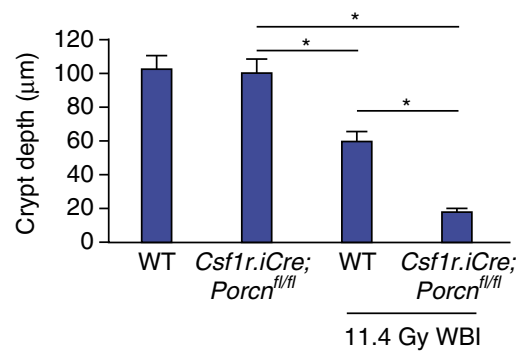

f

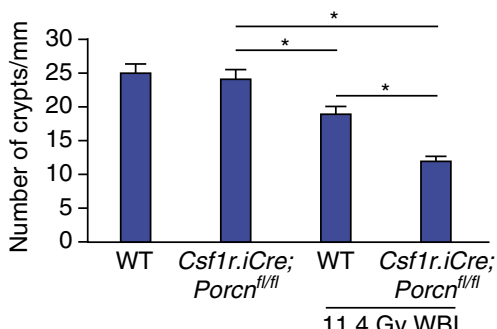

g

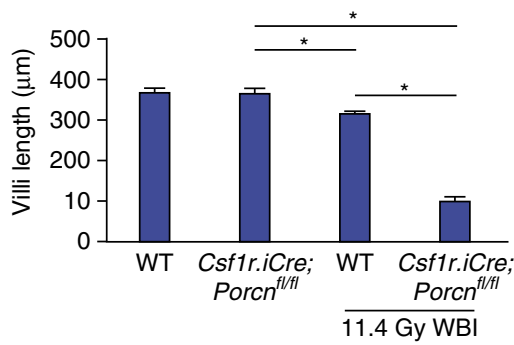


C59 a porcupine inhibitor ${ }^{1}$ also abolished WNT activity in $\mathrm{BMM} \phi \mathrm{CM}$ as determined by the TOPFLASH reporter assay. In contrast no activity was seen in the control FOPFLASH assay (mutant TCF/LEF reporter) indicating specificity for WNTs in the TOPFLASH assay. These data indicate that ablation of the Porcn floxed allele causes the absence of macrophage-derived WNT in BMM $\phi \mathrm{CM}$ and is similar to the pharmacological inhibition of Porcupine in macrophages. Since the population size of intestinal pericryptal macrophages is not sufficient to culture and obtain CM throughout the present study we have used BMM $\phi$ CM from WT and Porcn-deficient mice to determine the functional role of macrophage-derived WNTs.

Before determining the effect on radiation-induced repair, we assessed whether genetic ablation of Porcn in macrophages had significant consequences for mice and their intestinal development. Csf1r.iCre;Porcn flfl mice appeared phenotypically normal, having similar lifespans (followed up to 18 months of age), and intestinal morphology (Supplementary Fig. 2a), compared with WT. The total number of intestinal macrophages $\left(\mathrm{CD} 11 \mathrm{~b}^{+\mathrm{ve}} \mathrm{CX} 3 \mathrm{CR} 1^{+ \text {ve }} \mathrm{F} 480^{+\mathrm{ve}}\right.$; Supplementary Fig. 2b) were similar in Csflr.iCre; Porcn fl/fl and WT with/without irradiation indicating that Porcn deletion does not influence the population size of intestinal macrophages.

Mortality from acute radiation syndrome results from dose-dependent radiation injury to various organs ${ }^{2,9,33}$. Female Csf1r.iCre;Porcn fl/fl and WT mice were exposed to WBI and observed over 60 days for survival. No significant survival difference between Csf1r.iCre;Porcn fl/fl or WT mice was observed with the dose of 9.4-10.4 Gy $(P<0.9$ and $P<0.07$, respectively). However, with higher radiation doses (11.4-12.4 Gy WBI) Csflr.iCre;Porcn fl/fl mice demonstrated significantly reduced survival, with $100 \%$ mortality within $7-12$ days of WBI, compared with the WT cohort having $60 \%$ survival beyond 15 days post WBI (Fig. 1a, $P<0.0001, P<0.004$ Log-rank (Mantel-Cox) test).

The time to death after lethal radiation from haematopoietic failure and RIGS is dose-dependent. Moreover, ionizing radiation induces GI syndrome independent of bone marrow syndrome ${ }^{34}$. A WBI exposure of $>10.4 \mathrm{~Gy}$ within 5-14 days results in a characteristic RIGS, comprising of diarrhoea, weight loss and death. However, mortality at later time points (for example, $25-30$ days) post irradiation $(<10.4 \mathrm{~Gy})$ is attributed to the radiation-induced haematopoietic syndrome. In the present study, there was $100 \%$ mortality in Csf1r.iCre;Porcn flffl mice within 7-12 days of WBI of 11.4-12.4 Gy (Fig. 1a), suggesting their susceptibility to RIGS compared with the WT littermates. This conclusion was also supported by the observation of a reduction of body weight in the Csflr.iCre;Porcn ${ }^{f l f l}$ mice treated with $11.4 \mathrm{~Gy}$ by day 7 that was not observed in the WT littermates (Fig. 1b). However, the onset of mortality was at much later time points in Csflr.iCre;Porch flfl mice or their WT littermates exposed to $\leq 10.4 \mathrm{~Gy}$ (Fig. 1a) indicating that RIGS may not be the primary cause of death in these animals. These results further indicate that Csflr.iCre;Porcn fl/fl mice were more sensitive than WT controls to a radiation dose level that primarily induces RIGS.

Consistent with the effect of macrophage WNT loss enhancing RIGS and not effecting haematopoiesis, was that blood counts designed to assess haematopoietic syndrome did not show any differences among Csf1r.iCre;Porcn fl/fl mice and their WT littermate exposed to radiation (11.4-12.4 Gy; Fig. 1c). However, the histology of intestinal (jejunal) sections from irradiated mice showed more loss of crypt villus structure in Csf1r.iCre;Porcn fl/fl mice with significant reduction in Crypt depth, villi length and number of crypts compared with their WT controls (Crypt depth ${ }^{\star} P<8.84 \mathrm{E}-07$, villi length ${ }^{\star} P<7.98 \mathrm{E}-08$ and number of crypt ${ }^{\star} P<9.79 \mathrm{E}-09$ unpaired $t$-test, two-tailed; Fig. $\left.1 \mathrm{~d}-\mathrm{g}\right)$.

Porcn deletion in $\mathrm{M} \phi$ sensitize gut to Abdominal Irradiation. Since the effects of $10.4 \mathrm{~Gy}$ of WBI are secondary to combined haematopoietic and RIGS, we examined the effects of conditional Porcn deletion in myeloid cells on isolated RIGS injury by administering escalating doses of Abdominal Irradiation (AIR) after shielding the thorax, head and neck, and extremities, thus protecting the bone marrow ${ }^{2,9}$ (Fig. 2a). A single fraction of 18-20 Gy AIR was lethal in $100 \%$ of Csf1r.iCre;Porcn ${ }^{f l / f l}$ mice by 7-12 days. In contrast, $40 \%$ of WT littermates survived beyond 20 days post 18 Gy AIR $(P<0.009$, Log-rank (Mantel-Cox) test; Fig. 2b). Histopathology of jejunal sections at day 5 post AIR showed greater damage of the crypt villus structure with significant reduction in crypt depth $(P<9.64 \mathrm{E}-08)$, villi length $(P<7.8$-E07) and number of crypts $(P<8.77 \mathrm{E}-09$ (unpaired t-test, two-tailed) in Csf1r.iCre; Porcn ${ }^{f l f l}$ mice compared with their WT littermate (Fig. 2c-f). As the major part of the bone marrow was shielded, bone marrow was not severely damaged by $\mathrm{AIR}^{2}$, and therefore the observed mortality of the Csf1r.iCre;Porcn fl/fl mice was due to RIGS. Altogether these data clearly indicate that inhibition of myeloid-derived WNT release in Csf1r.iCre;Porcn fl/fl mice primarily increases gastrointestinal radio-sensitivity and facilitates the onset of RIGS.

Figure 1 | Deletion of Porcn in macrophages radio-sensitizes mice to lethal doses of WBI. (a) Kaplan-Meier survival analysis of $C s f 1 r . i C r e ; P o r c n^{f l / f l}$ and WT mice exposed to WBI (9.4-12.4 Gy). Csf1r.iCre;Porcn ${ }^{f l / f l}$ mice show reduced survival following lethal doses of WBI (11.4-12.4 Gy WBI) with $100 \%$ mortality within 7-12 days of radiation exposure, compared with WT that have $60 \%$ survival beyond 15 days post WBI $(P<0.0001, P<0.004$ Log-rank (Mantel-Cox) test; $n=15$ per group). No significant survival difference between Csf1r.iCre;Porc $n^{f / f f l}$ or WT littermate mice was observed with the irradiation dose of 9.4-10.4 Gy WBI ( $n=15$ per group). (b) Body weight of mice at post irradiation time points (11.4 Gy and 12.4 Gy WBI). (c) Complete blood count (CBC) analysis. CBC measurements for Csf1r.iCre; Porcn ${ }^{f / f l}$ and WT mice exposed to $0 \mathrm{~Gy} / 11.4 \mathrm{~Gy} / 12.4 \mathrm{~Gy}$ WBI. Blood samples were drawn at 5 days post irradiation ( $n=3$ per group). (d) HE staining of jejunum section from Csf1r.iCre;Porcn ${ }^{f l / f l}$ and WT mice exposed to 0 Gy/11.4 Gy WBI at day 5 post irradiation. No significant differences in crypt villus structure were noted in non-irradiated Csf1r.iCre;Porcn ${ }^{f / / f l}$ mice compared with WT mice. Note, shortening of crypt depth as well as loss of crypts in Csf1r.iCre;Porc $n^{f l / f l}$ mice exposed to irradiation ( $n=3$ per group) compared with WT mice. (e) Histogram demonstrating crypt depth $(\mu \mathrm{M})$ from jejunal sections of Csf1r.iCre; Porcn ${ }^{f l / f l}$ and WT mice exposed to 0 or $11.4 \mathrm{~Gy}$ WBI. Both WT and Csf1r.iCre; Porcn ${ }^{f / / f l}$ mice exposed to irradiation showed reduction in crypt depth at day 5 post irradiation compared with un-irradiated control WT mice ${ }^{\star} P<4.66 \mathrm{E}-05$, Csf1riCre; Porcn ${ }^{f l / f l}$ mice ${ }^{\star} P<9.63 \mathrm{E}-08$. However, loss of crypt depth was significantly greater in Csf1riCre;Porcn ${ }^{f / f l}$ mice compared with $\mathrm{WT}$ mice ${ }^{\star} P<8.84 \mathrm{E}-07$ unpaired $t$-test, two-tailed. (f) Histogram demonstrating the number of crypts $\mathrm{mm}^{-1}$ from jejunal section of Csf1r.iCre;Porc $n^{f l / f l}$ and WT mice exposed to 0 or $11.4 \mathrm{~Gy}$ WBI. Both WT and Csf1riCre; Porcn ${ }^{f / / f l}$ mice exposed to irradiation showed reduction in crypt number at day 5 post irradiation compared with un-irradiated control WT mice ${ }^{\star} P<0.0008, C s f 1$ r.iCre; Porc $n^{f l / f l}$ mice ${ }^{\star} P<1 \mathrm{E}-08$. However, loss of crypt was significantly greater in $C s f 1 r . i C r e ; P o r c n^{f l / f l}$ mice compared with WT mice ${ }^{*} P<9.79 \mathrm{E}-09$ unpaired $t$-test, two-tailed. (g) Histogram demonstrating villi length from jejunal section of Csf1r.iCre; Porcn ${ }^{f l / f l}$ and WT mice exposed to 0 or $11.4 \mathrm{~Gy}$ WBI. Both WT and Csf1riCre;Porch ${ }^{f l / f l}$ mice exposed to irradiation showed reduction in crypt number at day 5 post irradiation compared with un-irradiated control WT mice ${ }^{\star} P<0.0006, C s f 1 r i$ Cre; $P$ orcn ${ }^{f l / f l}$ mice ${ }^{\star} P<8.9 \mathrm{E}-08$. However, loss of villi length was significantly greater in Csf1r.iCre;Porcn ${ }^{f l / f l}$ mice compared with WT mice ${ }^{\star} P<7.98 \mathrm{E}-09$ unpaired $t$-test, two-tailed. 
a

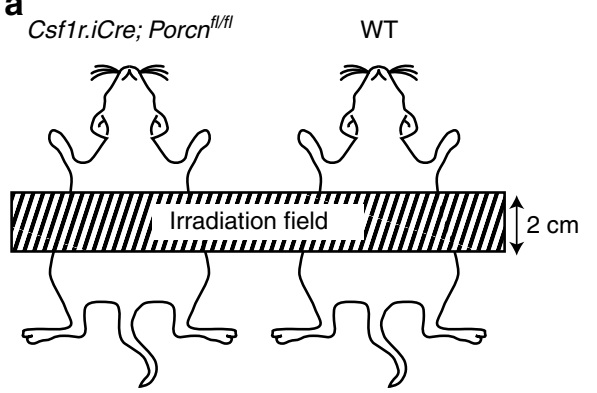

C
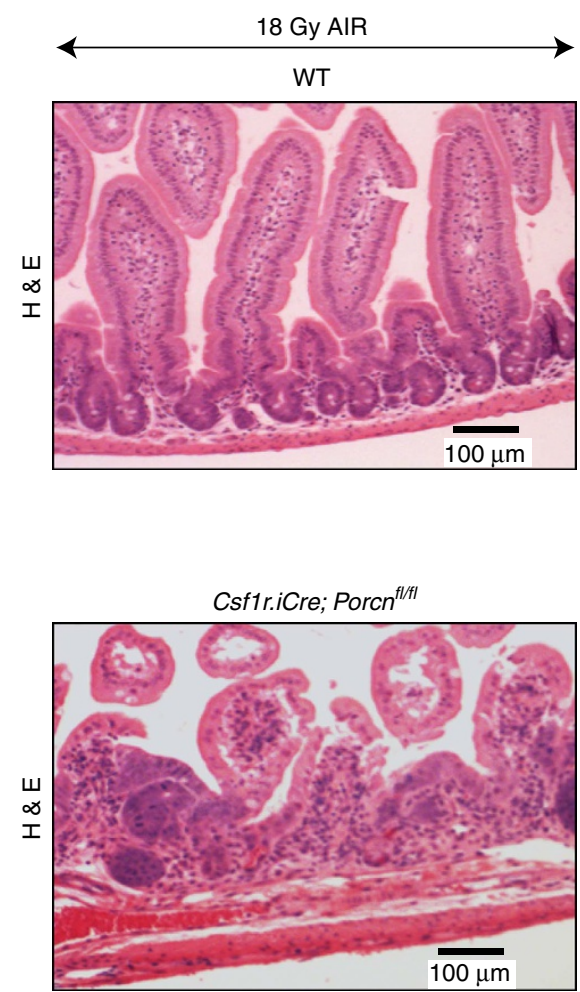

b

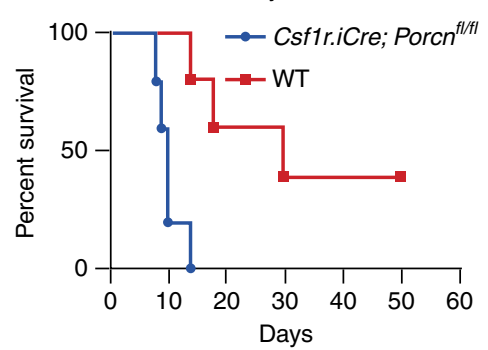

d
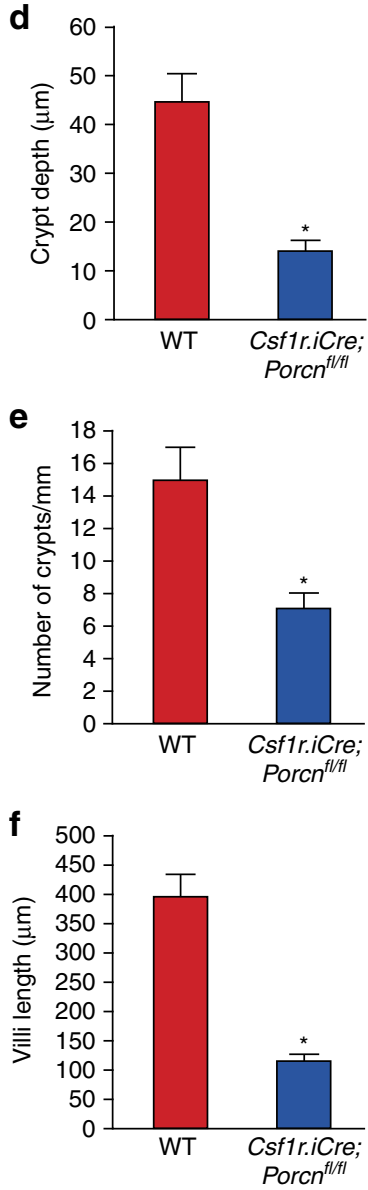

Figure 2 | Deletion of Porcn in macrophages sensitizes mice against lethal dose of AIR. (a) Schematic diagram demonstrating the AIR exposure field for Csf1riCre;Porcn ${ }^{f l / f l}$ and WT mice. A $2 \mathrm{~cm}$ area of the mice containing the GI was irradiated (irradiation field), thus shielding the upper thorax, head and neck as well as lower and upper extremities, protecting a significant portion of the bone marrow, thus inducing predominantly RIGS. (b) Kaplan-Meier survival analysis. Csf1r.iCre;Porc $n^{f / / f l}$ mice have reduced survival against a lethal dose (18 Gy) of abdominal radiation compared with WT mice ( $n=10$ per group; $P<0.009$ Log-rank (Mantel-Cox) test). (c) HE staining of jejunum sections from Csf1r.iCre;Porct ${ }^{f / f f l}$ and WT mice exposed to 18 Gy AIR. Mice were killed and jejunum was collected at day 5 post irradiation. Csf1r.iCre;Porcn ${ }^{f l / f l}$ mice showed more villi denudation and crypt loss compared with WT littermate mice at day 5 post irradiation ( $n=3$ per group). (d) Histogram showing crypt depth $\left(\mu M\right.$ ) in jejunal sections of Csf1riCre;Porc ${ }^{f l / f l}$ and WT mice exposed to 0 Gy or 18 Gy AIR. Csf1riCre;Porcn ${ }^{f / / f l}$ mice exposed to AIR had significantly higher reduction in crypt depth compared with WT ${ }^{*} P<9.64 \mathrm{E}-08$ unpaired $t$-test, two-tailed. (e) Histogram showing number of crypts $\mathrm{mm}^{-1}$ in jejunal sections of Csf1r.iCre;Porch ${ }^{f / f l}$ and WT mice exposed to $0 \mathrm{~Gy}$ or $18 \mathrm{~Gy}$ AIR.

Csflr.iCre;Porcn ${ }^{f l / f l}$ mice exposed to AIR has significantly higher reduction in crypt number compared with WT ${ }^{\star} P<8.77 \mathrm{E}-09$ unpaired $t$-test, two-tailed. (f) Histogram showing villus length in jejunal sections of Csf1riCre; Porcn fl/fl and WT mice exposed to 0 or $18 \mathrm{~Gy}$ AIR. Csf1r.iCre;Porcn ${ }^{f l / f l}$ mice exposed to AIR has significantly higher reduction in villi length compared with $\mathrm{WT}{ }^{\star} P<8 \mathrm{E}-07$ unpaired $t$-test, two-tailed.

WT but not Porcn-null BMMФ CM treatment inhibits RIGS. Since WT BMMФ CM had shown higher WNT activity compared with CM from porcupine-null BMMФ (Supplementary Fig. 1e), we examined whether BMMФ CM could rescue Csflr.iCre;Porcn flfl mice from RIGS. Csf1r.iCre;Porcn fl/fl mice exposed to WBI (11.2 Gy) were treated with CM (i.v.) derived from WT or Porcn-null BMMФ at $1 \mathrm{~h}$ and $24 \mathrm{~h}$ post WBI. Animals only exposed to $11.2 \mathrm{~Gy}$ WBI or receiving porcupine-null BMM $\Phi \mathrm{CM}$ after radiation died within 12 days of radiation exposure (Fig. 3a) with characteristic signs and symptoms of RIGS, including diarrhoea, black stools and weight loss. In contrast, animals that received WT BMMФ CM had 
well-formed stools, maintained body weight and had $40 \%$ survival beyond 20 days $(P<0.01$, Log-rank (Mantel-Cox) test; Fig. 3a).

Histopathological analysis of jejunal section at 3.5 days post WBI showed crypt depletion and a decrease in crypt regeneration followed by villi denudation in Csflr.iCre;Porcn fl/fl mice
(Fig. 3b,d,e). Treatment with Porcn-null BMMФ CM failed to rescue the crypt villus structure following irradiation (Fig. 3b,d,e). However, CM from WT BMMФ improved the overall crypt villus architecture in irradiated Csf1r.iCre;Porcn fl/fl mice, with an increase in number of crypts and preserved villous length

a
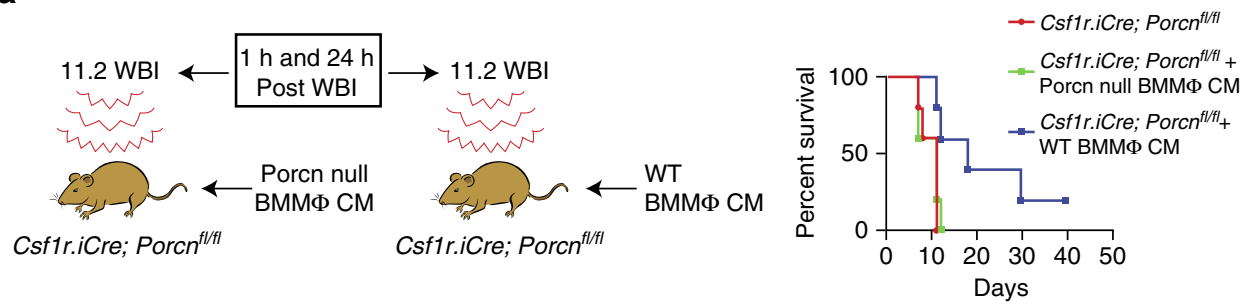

b
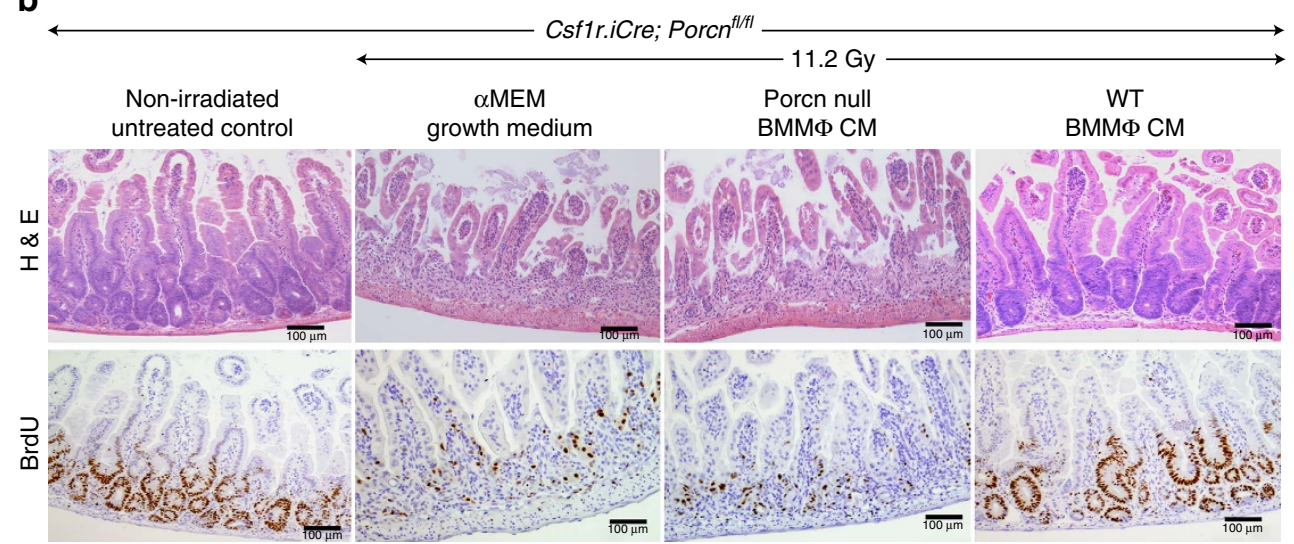

C

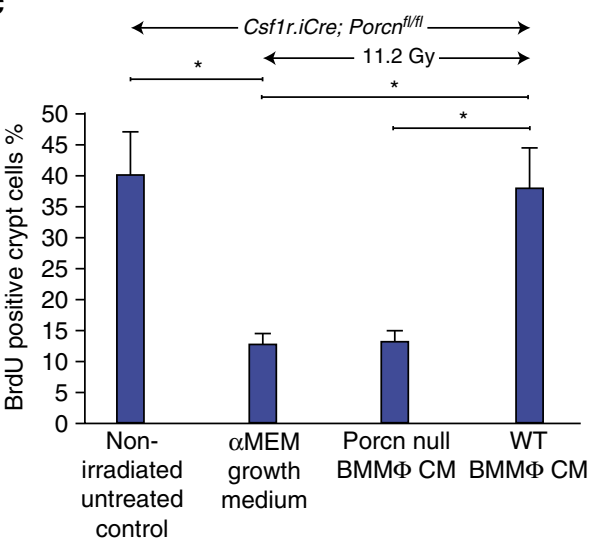

e

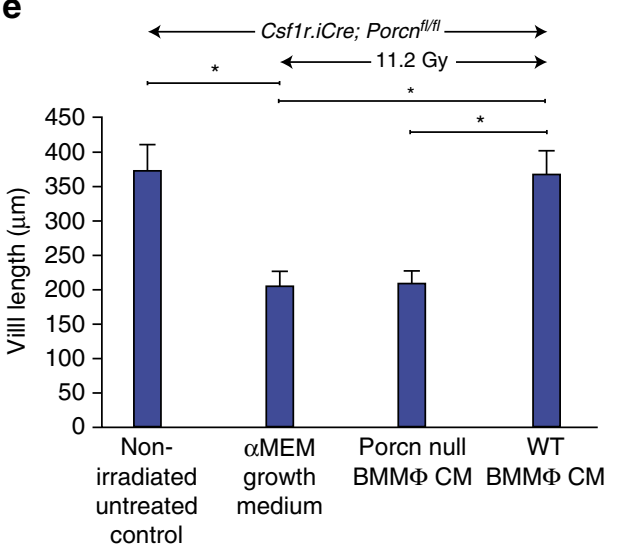

d

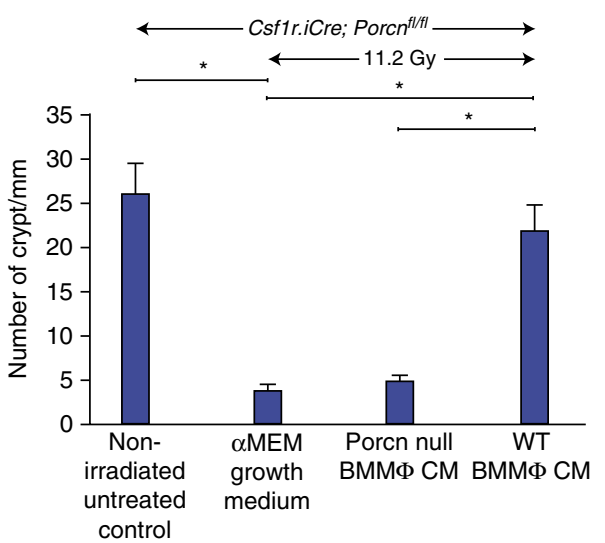

f

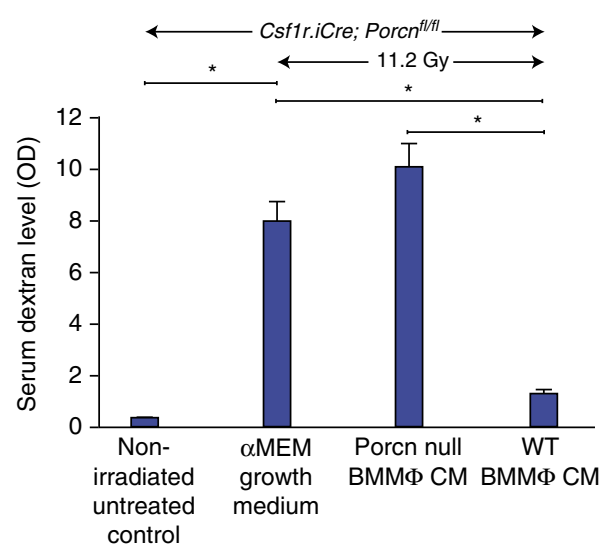


(Fig. 3b,d,e). The percentage of the $\mathrm{BrdU}^{+}$ve crypt epithelial cells synthesizing DNA was significantly enhanced in WT cohort at 3.5 days post irradiation $(P<2.21 \mathrm{E}-07$ unpaired $t$-test, two-tailed; Fig. 3b,c). However, treatment with CM from WT or Porcn-null BMMФ in non-irradiated mice did not induce any changes in crypt villus morphology and BrdU incorporation (Supplementary Fig. 3).

Since dextran is unable to cross the GI epithelia unless it is compromised, dextran in the blood is a good indicator of epithelial damage ${ }^{35}$, blood FITC-dextran levels were measured at $4 \mathrm{~h}$ after gavage. Treatment with WT ВMMФ CM significantly reduced the FITC-dextran uptake in the blood stream in irradiated Csf1r.iCre;Porcn fl/fl mice compared with mice receiving Porcn-null BMMФ CM $(P<0.007$ unpaired $t$-test, two-tailed; Fig. 3f). These data indicate restitution of intestinal epithelial integrity by ВММФ СМ.

Csf1r.iCre;Porcn fl/fl mice exposed to AIR (18 Gy) were rescued with the WT ВММФ CM treatment with $60 \%$ mice surviving beyond 20 days of exposure $(P<0.002$ Log-rank (Mantel-Cox) test; Fig. 4a,b). In contrast, Csf1r.iCre;Porcn ${ }^{f l f l}$ mice exposed to AIR and receiving Porcn-null BMMФ CM died within 10-12 days post AIR similar to irradiated untreated control. Histopathological evidence clearly indicates that RIGS is the primary cause of death in Porcn-null BMMФ CM-treated animals as there was a significant loss of crypt and villus denudation (Fig. 4c-e). However, irradiated mice receiving WT ВMМФ CM had a significant restitution of crypt villus structure (Fig. 4c-e). These results indicate that inhibition of BMM $\Phi$ derived WNT in the $\mathrm{CM}$ is critical for regenerative response against RIGS. However, further optimization of CM treatment is needed for complete recovery against RIGS induced by lethal dose of AIR.

EV depletion reverses radio-mitigating role of WT ВММФ СМ. EVs are involved in the secretion of WNT proteins and have been shown to transmit morphogen signalling ${ }^{36}$. It has also been reported that depletion of EVs in cell culture supernatant reduces WNT activity ${ }^{37}$. Considering the hydrophobic properties of $\mathrm{WNTs}^{38,39}$, their normal short half-life in serum ${ }^{37}$ and their paracrine action in the ВММФ CM treatment experiments shown here, we examined whether WNTs are secreted in EVs protecting them from degradation. We first depleted EV from WT BMMФ $\mathrm{CM}$ to reduce the WNT activity and therefore ablate the radiomitigating role of ВММФ CM treatment. Depletion of EV from WT ВMMФ CM was confirmed by the absence of TSG101, ALIX and CD9 positive band that is diagnostic of $\mathrm{EVs}^{37}$ in the depleted fraction (Supplementary Fig. 4a). TCF/LEF reporter assay demonstrated that depletion of EVs removed the WNT activity from WT ВММФ CM (Supplementary Fig. 4b) which was recovered with add-back of the purified EV fraction to the depleted fraction (Supplementary Fig. 4b). Concentration of the EV fraction was required to recover activity probably because the EV preparation was not optimized to maintain WNT activity. We also confirmed that absence of WNT activity in the depleted fraction is not due to adsorption of non-EV-packaged WNT by the EV depletion as addition of recombinant WNT6 or WNT9a $\left(1 \mu \mathrm{g} \mathrm{ml}^{-1}\right)$ before EV depletion showed WNT activity in EV-depleted fraction (Supplementary Fig. 4b).

Treatment with the EV-depleted WT BMMФ CM could not rescue Csf1r.iCre;Porcn fl/fl mice exposed to 18 Gy AIR (Fig. 4b) or WT mice exposed to $18.5 \mathrm{~Gy}$ AIR (Fig. 5a) from radiation lethality as $80-100 \%$ of animals were dead within $10-12$ days of radiation exposure compared with the significantly enhanced survival of mice treated with WT CM $(P<0.002$ Log-rank (Mantel-Cox) test; Fig. 4b and Fig. 5a).

We also purified the EVs from WT/Porcn-null BMMФ CM using conventional ultra-centrifugation methods. EV positive for TSG101, ALIX and CD9 (Fig. 5b) demonstrated WNT activity only when purified from WT BMMФ CM but not from Porcn-null BMМФ CM (Fig. 5c). Furthermore, the WNT activity (Fig. 5c) and radioprotective activity (Fig. 5a) was entirely removed from the EV-depleted CM effluent. Using an enzymelinked immunosorbant assay (ELISA) specific for individual WNTs the presence of WNT5a, WNT6 and WNT9a were detected in EVs purified from WT ВMMФ CM (Fig. 5d-f). These data, using two methods of EV purification, indicate that functional WNT ligands are secreted into BMMФ CM as EV-packaged proteins and these are responsible for the radioprotective effect.

MФ-derived WNTs activate $\beta$-catenin in irradiated crypt. Secretory WNTs induce nuclear translocation and activation of $\beta$-catenin to drive a gene-expression programme that supports stem cell maintenance, proliferation. Immunohistochemical analysis of jejunal sections from non-irradiated Csf1r.iCre;Porcn flffl mice showed characteristic nuclear $\beta$-catenin staining with $39 \pm 2$ of cells being positive for nuclear $\beta$-catenin (per 75 crypts; Fig. $6 a, b)$. Similar patterns of nuclear $\beta$-catenin staining were also observed in WT mice (Supplementary Fig. 5). Irradiated Csf1r.iCre;Porcn fl/fl mice treated with alpha minimal essential medium $(\alpha M E M)$ or CM form Porcn-null BMMФ had significantly fewer nuclear $\beta$-catenin-positive cells $(15 \pm 2.2$ and $22 \pm 2$, respectively) compared with non-irradiated untreated control $(P<1 \mathrm{E}-04$ and $P<1.2 \mathrm{E}-04$, respectively unpaired $t$-test, two-tailed). However, treatment with WT BMMФ CM

Figure 3 | CM from WT but not from Porcn-null BMMФ inhibits RIGS in Csf1r.iCre;Porcn ${ }^{\text {fl/fl }}$ mice exposed to lethal dose of WBI. (a) Experimental design and Kaplan-Meier survival analysis of Csf1riCre;Porcn ${ }^{f / f l}$ mice ( $n=10$ per group) receiving CM (i.v.) derived from WT/Porcn-null BMM 1 at 1 and $48 \mathrm{~h}$ post WBI (11.2 Gy). Mice receiving WT BMMФ CM showed $40 \%$ survival ( $P<0.003$ Log-rank (Mantel-Cox) test) beyond 25 days compared with mice receiving Porcn-null BMМФ CM or untreated mice where $100 \%$ of mice died within 12 days after irradiation. (b) Representative HE and BrdU immunohistochemistry of mice jejunal sections. Note, restitution on crypt villus structure with the increase in crypt cell proliferation in Csf1r.iCre;Porcn ${ }^{f l / f l}$ mice receiving WT ВMМФ CM compared with Porcn-null BMMФ CM treatment. (c) The proliferation rate was calculated as the percentage of BrdU-positive cells over the total number of cells in each crypt and displayed as bar diagrams. Crypt cell proliferation rate in irradiated mice: WT BMM $\Phi$ $\mathrm{CM}$ versus Porcn-null BMMФ CM treatment group ${ }^{\star} P<2.21 \mathrm{E}-07$ ( $n=5$ per group), WT BMM $\Phi$ CM versus $\alpha M E M$ growth medium treatment group ${ }^{\star} P<2.69 \mathrm{E}-07$ ( $n=5$ per group; unpaired $t$-test, two-tailed). (d) Histogram showing number of crypts mm ${ }^{-1}$ in jejunal sections of $C s f 1$ r.iCre;Porc $n^{f l / f l}$ mice. Irradiated Csf1riCre;Porcn ${ }^{f l / f l}$ mice receiving WT BMMФ CM showed less crypt loss compared with mice receiving Porcn-null BMMФ CM or $\alpha M E M$ growth medium $\left({ }^{\star} P<6.86 \mathrm{E}-09\right.$ and ${ }^{\star} P<6.74 \mathrm{E}-08$ unpaired $t$-test, two-tailed). (e) Histogram showing villus length in jejunal sections of $C s f 1 r . i C r e ; P o r c n^{f l / f l}$ mice. Irradiated Csf1riCre;Porcn ${ }^{f l / f l}$ mice receiving WT BMMФ CM showed less reduction in villi length compared with mice receiving Porcn-null BMMФ CM or $\alpha$ MEM growth medium $\left({ }^{\star} P<3.74 \mathrm{E}-06\right.$ and ${ }^{\star} P<3.60 \mathrm{E}-06$ unpaired $t$-test, two-tailed). (f) Histogram demonstrating serum dextran level in Csf1r.iCre;Porcn ${ }^{f / f l}$ mice. Mice receiving WT BMМФ CM showed a lower serum dextran level thereby suggesting restitution of epithelial integrity compared with mice receiving Porcn-null BMMФ CM or $\alpha$ MEM growth medium ( ${ }^{\star} P<0.006$ and ${ }^{\star} P<0.009$ respectively; $n=3$ per group). Untreated mice also showed a lower serum dextran level compared with irradiated mice receiving $\alpha$ MEM growth medium $(P<0.003)$ or $P$ orcn-null BMM $1 \mathrm{CM}(P<0.001$; unpaired $t$-test, two-tailed). 
significantly increased the number of nuclear $\beta$-catenin-positive crypt epithelial cells $(38 \pm 1.2)$ compared with $\alpha \mathrm{MEM}$ or Porcn-null BMMФ CM-treated cohort $(P<1 \mathrm{E}-04$ and $P<1 \mathrm{E}-04$, respectively, unpaired $t$-test, two-tailed; Fig. 6a,b).
Consistent with our immunohistological analysis of nuclear $\beta$-catenin expression, the real-time PCR array analysis of $\beta$-catenin target genes in crypt epithelial cells showed several-fold increases in mRNA level in irradiated mice treated a

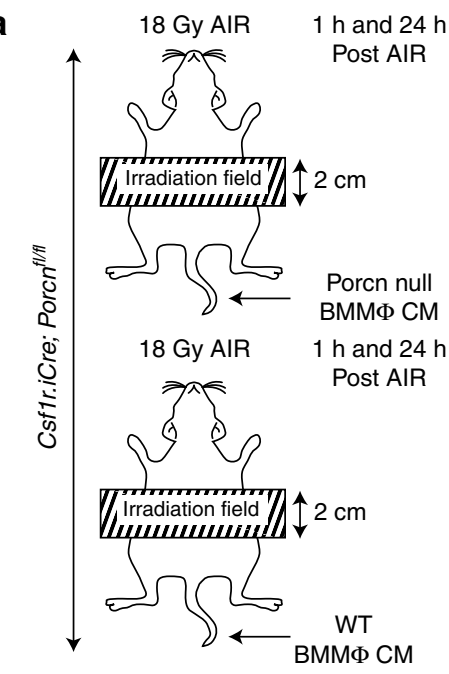

b

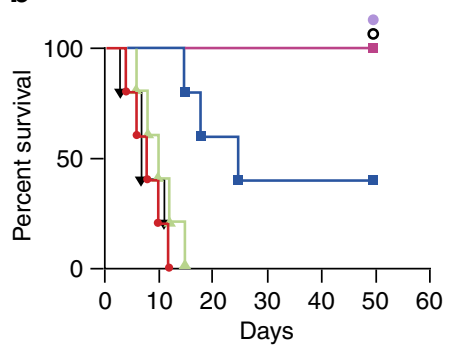

$\rightarrow 0$ Gy AIR + EV reagent

$-0-0$ Gy AIR + WT BMMФ CM

$\rightarrow-0$ Gy AIR + Porcn null BMM $\Phi$ CM

$\rightarrow 18$ Gy AIR

-18 Gy AIR + Porcn null BMMФ CM

$\rightarrow 18$ Gy AIR + WT BMMФ CM

$\rightarrow 18$ Gy AIR + EV depleted WT BMMФ CM
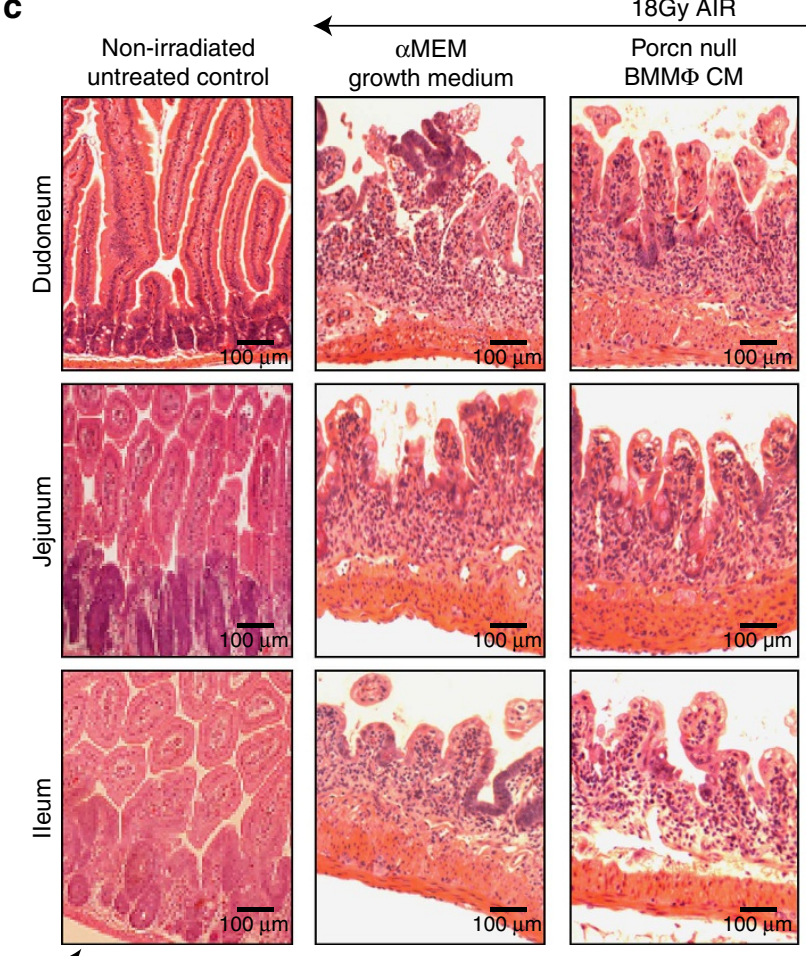

18Gy AIR

$\begin{array}{cc}\text { Porcn null } & \text { WT } \\ \text { ВМMФ CM } & \text { ВMMФ CM }\end{array}$
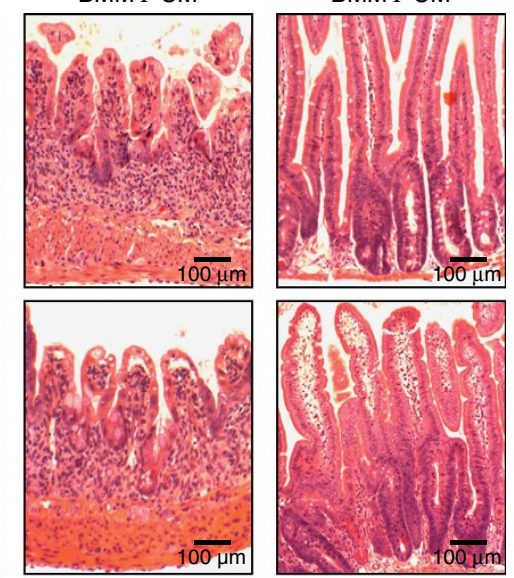

Csf1r.iCre; Porch ${ }^{f / f t}$
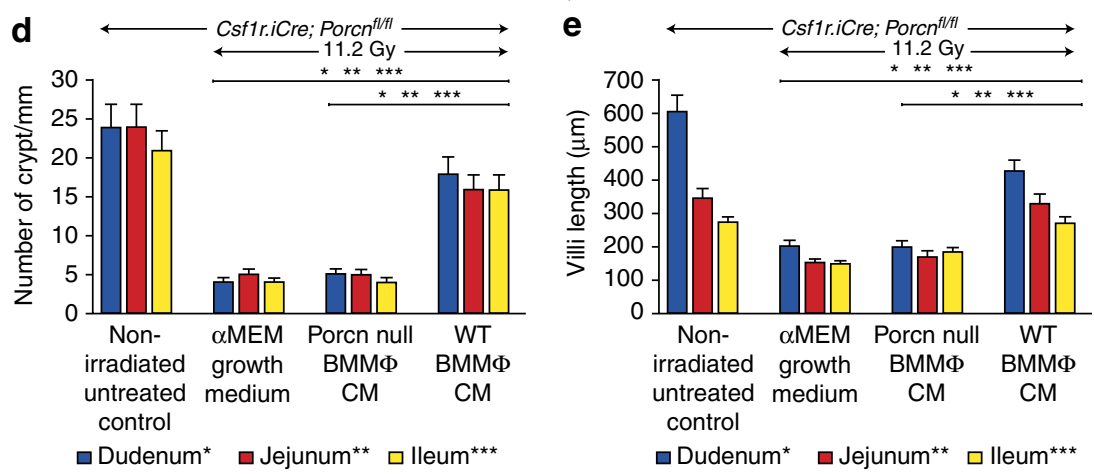
with WT ВMМФ CM compared with those mice receiving Porcn-null ВММФ CM (Table 1). Altogether these data indicate that irradiated crypts and that this WNT signal is needed to stimulate proliferation and crypt regeneration.

BMMФ WNTs rescue Lgr5 ${ }^{+ \text {ve }}$ ISC from radiation toxicity. To analyse further the specific roles of macrophage-derived WNTs we turned to an in vitro primary intestinal organoid culture system exposed to graded doses of radiation. Intestinal crypts were isolated from the Lgr5/GFP-IRES-Cre-ERT2 knock-in mice to allow visualization of the ISCs and these were cultured in vitro as organoids. Irradiation of these intestinal organoids with 2-6 Gy resulted in the loss of budding crypt in a dose-dependent manner (Supplementary Fig. 6). At a radiation dose level of $8 \mathrm{~Gy}$, most of the Lgr5 ${ }^{+ \text {ve }}$ ISCs had disappeared within $48 \mathrm{~h}$ post irradiation (Fig. 7a, top panel), resulting in a significant loss of budding crypts with changes in existing crypt morphology indicating inhibition of ISC growth and differentiation in response to radiation exposure.

We next examined the effect of macrophage $\mathrm{CM}$ on these crypt organoids exposed to 4-8 Gy irradiation. At every dose of radiation $\mathrm{CM}$ from WT ВMM $\Phi$ rescued the organoids from radiation toxicity and restored the number of budding crypts to untreated levels as represented by ratio of number of budding crypt/total crypt (Fig. 7a,b). In contrast Csfir.iCre;Porcn ${ }^{\text {fl/fl }} \mathrm{CM}$ $B M M \Phi$ failed to rescue organoids from radiation damage (Fig. 7a,b). A representative example of the effects of WT BMMФ $\mathrm{CM}$ on budding crypt having Lgr5 ${ }^{+\mathrm{ve}}$ ISCs $\left(\mathrm{GFP}^{+\mathrm{ve}}\right.$ indicated with arrow) is shown in Fig. 7a. In these microscopic observations over many experiments and analysis of crypts we observed that CM from WT ВMMФ CM but not Porcn-null ВМMФ CM rescued Lgr5 ${ }^{+ \text {ve }}$ ISCs from 8 Gy radiation toxicity. Thus in the case of Porcn-null ВMМФ СM most and usually all of the Lgr5 ${ }^{+\mathrm{ve}} \mathrm{GFP}^{+\mathrm{ve}}$ cells at the base of the budding crypt had disappeared within $48 \mathrm{~h}$ of radiation exposure (Fig. 7a).

To study this effect in vivo we examined the role of macrophage-derived WNT on ISC survival by exposing Lgr5/GFP-IRES-Cre-ERT2 knock-in mice to $10.4 \mathrm{~Gy} \mathrm{WBI}$ and then treated with CM (i.v.) from WT BMMФ or Porcn-null BMM $\Phi$ at $1 \mathrm{~h}$ and $24 \mathrm{~h}$ post WBI. LGR5 ${ }^{+\mathrm{ve}}$ ISCs had disappeared from the crypt base within $48 \mathrm{~h}$ of radiation in mice receiving either $\alpha$ MEM growth medium or CM from Porcn-null ВMMФ (Fig. 7c,d). However, mice receiving WT ВMMФ CM showed significant preservation of LGR5 ${ }^{+\mathrm{ve}}$ ISCs beyond $48 \mathrm{~h}$ post WBI (Fig. $7 \mathrm{c}$,d; $P<0.0002$ compared with mice receiving CM from Porcn-null BMM $\Phi$ and $P<1.75 \mathrm{E}-06$ compared with irradiated control receiving $\alpha$ MEM growth medium, respectively, unpaired $t$-test, two-tailed). These results clearly indicate that macrophage-derived WNTs are critical for self-renewal and proliferation of ISCs following radiation in vivo and in vitro, and that inhibition of macrophage-derived Wnt secretion via deletion of Porcn impaired the regenerative response of ISC following radiation toxicity.

Identification of MФ WNTs protecting ISC against irradiation. To identify the WNTs responsible for crypt regeneration we first determined those expressed by BMM $\Phi$ and intestinal macrophages then used an add-back system to the Porcn-null BMM $\Phi$ CM that has no WNTs in the organoid cultures to determine their function. RNA was isolated from BMM $\Phi$ or flow-sorted intestinal macrophages and subjected to qRT-PCR analysis. This analysis indicated that Wnt5a, Wnt6 and Wnt9a were the only WNTs expressed in BMM $\Phi$ (Fig. 7e). WNT5a, WNT6 and WNT9a were also predominantly expressed in $\mathrm{CD}_{11 \mathrm{~b}^{+}}$ ${ }^{\mathrm{ve}} \mathrm{F} 480^{+\mathrm{ve}} \mathrm{CX} 3 \mathrm{CR} 1{ }^{+\mathrm{ve}} \mathrm{Ly} 6 \mathrm{C}^{+\mathrm{ve}}$ intestinal macrophages and WNT6 in $\mathrm{CD}_{11} \mathrm{~b}^{+\mathrm{ve}} \mathrm{F} 480^{+\mathrm{ve}} \mathrm{CX} 3 \mathrm{CR} 1^{+\mathrm{ve}} \mathrm{Ly} 6 \mathrm{C}^{-\mathrm{ve}}$ intestinal macrophages (Fig. 7f). To determine whether these WNT ligands can mediate ISC regeneration following radiation, we examined their effects on the growth of intestinal organoids. Primary crypt cultures were first incubated with CM derived from Porcn-null BMM $\Phi$ following exposure to $6 \mathrm{~Gy}$ of irradiation and then treated with WNT ligands. At $48 \mathrm{~h}$ post irradiation, the number of budding crypts was counted and the ratio of budding crypts to total number of crypts determined. Treatment with all the expressed WNTs 5a, 6 and 9a showed significant improvement in organoid growth $(P<0.009, \quad P<0.0001$, $P<8.99662 \mathrm{E}-05$ respectively, unpaired $t$-test, two-tailed) compared with the irradiated control with WNT6 being the most efficient (Fig. $7 \mathrm{~g}$ ). Similar responses were noted when irradiated organoids were treated with WNT in absence of CM indicating that observed organoid growth is a direct effect of WNT supplement (Supplementary Fig. 7a). We have also examined whether the presence or absence of RSPO1 a WNT agonist in ВММФ СM is contributing to the observed effect of ВMMФ derived WNT in organoids as this has been reported to be involved in crypt repair ${ }^{9}$. RSPO1 was detected in both WT and Porcn-null ВММФ CM even though the latter cannot rescue crypt development after radiation (Supplementary Fig. 7b) indicating that the observed effect of ВMMФ $\mathrm{CM}$ in the organoid cultures is primarily due to presence of WNTs. WNT6 and WNT9a are canonical signalling molecules, while WNT5A can activate both canonical and non-canonical pathways depending on the presence of different receptors ${ }^{40,41}$ However, the crypt organoid study indicates that canonical WNT signalling plays a major role in organoid growth against radiation as non-canonical WNT5b failed to induce crypt regeneration (Fig. $7 \mathrm{~g}$ ). In the experiments described above, we also observed

Figure 4 | WNTs in BMMФ CM are required to inhibit RIGS in Csf1r.iCre;Porcn ${ }^{f l / f l}$ mice exposed to AIR. (a) Experimental design for partial body irradiation. Mice exposed to AIR were treated with WT or Porcn-null BMМФ CM at 1 and $24 \mathrm{~h}$ post exposure. (b) Kaplan-Meier survival analysis of

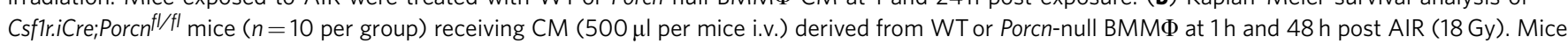
receiving WT BMMФ CM showed $60 \%$ survival beyond 20 days compared with mice receiving Porcn-null BMMФ CM or EV-depleted WT BMMФ CM, where $100 \%$ of mice died within 12 days after irradiation $(P<0.002$ and $P<0.003$, respectively, Log-rank (Mantel-Cox) test). Reagent used for chemical depletion of EV did not confer any toxicity to normal mice. (c) HE-stained representative transverse sections of duodenum, jejunum and ileum from Csf1r.iCre;Porcn ${ }^{f l / f l}$ mice ( $n=3$ per group). Note, restitution on crypt villus structure in irradiated Csf1r.iCre;Porcn ${ }^{f l / f l}$ mice receiving WT BMMФ CM. However, treatment with Porcn-null ВММФ CM or $\alpha$ MEM growth medium showed significant loss of crypts along with villi denudation. (d) Histogram demonstrating number of crypts $\mathrm{mm}^{-1}$ in duodenal, jejuna, and illeul sections of Csf1r.iCre;Porcn ${ }^{f l / f l}$ mice. Irradiated Csf1riCre;Porcn ${ }^{f l / f l}$ mice receiving WT BMMФ CM showed less crypt loss compared with mice receiving Porcn-null BMMФ CM (Duodenum ${ }^{\star} P<6.86 \mathrm{E}-07$, Jejunum ${ }^{\star \star} P<7.89 \mathrm{E}-08$ and Ileum ${ }^{\star \star \star} P<8.16 \mathrm{E}-08$ ) or $\alpha \mathrm{MEM}$ growth medium (Duodenum ${ }^{\star} P<7.92 \mathrm{E}-08$, Jejunum ${ }^{\star \star} P<8.26 \mathrm{E}-07$ and lleum ${ }^{\star \star \star} P<8.96 \mathrm{E}-09$; unpaired $t$-test, two-tailed). (e) Histogram demonstrating villus length in duodenal, jejunal and illeul sections of Csf1r.iCre;Porcn ${ }^{f l / f l}$ mice. Irradiated Csf1r.iCre; Porcn ${ }^{f / / f l}$ mice receiving WT BMMФ CM showed less reduction in villus length compared with mice receiving Porcn-null BMM $\mathrm{B} C M$ (Duodenum ${ }^{*} P<3.64 \mathrm{E}-06$, Jejunum ${ }^{\star \star} P<2.86 \mathrm{E}-06$ and Ileum ${ }^{\star \star \star} P<2.16 \mathrm{E}-05$ ) or $\alpha \mathrm{MEM}$ growth medium (Duodenum ${ }^{\star} P<4.21 \mathrm{E}-06$, Jejunum ${ }^{\star \star} P<3.16 \mathrm{E}-08$ and Ileum ${ }^{\star \star \star} P<2.88 \mathrm{E}-05$; unpaired $t$-test, two-tailed). 
a

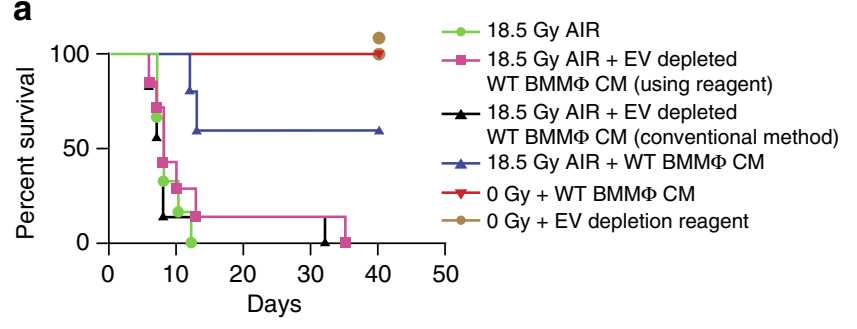

b

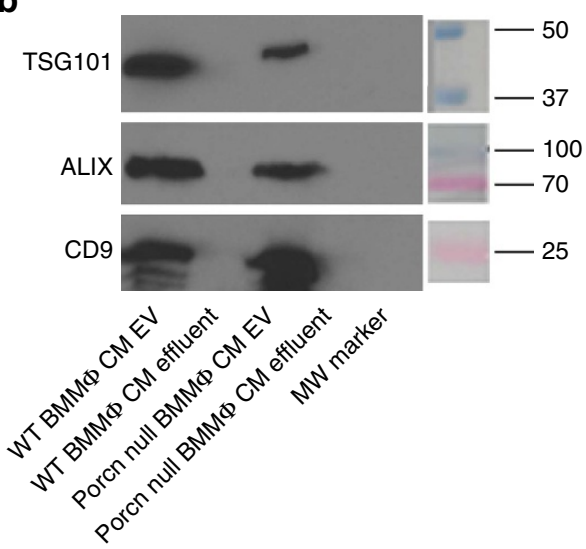

C

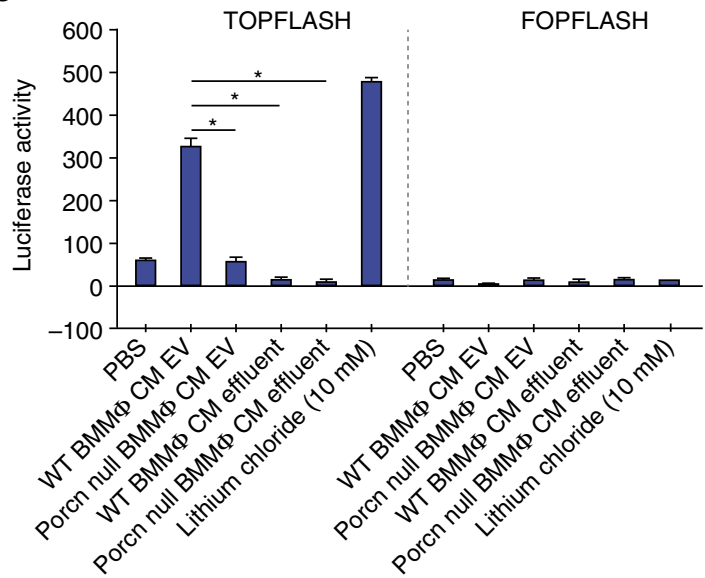

d

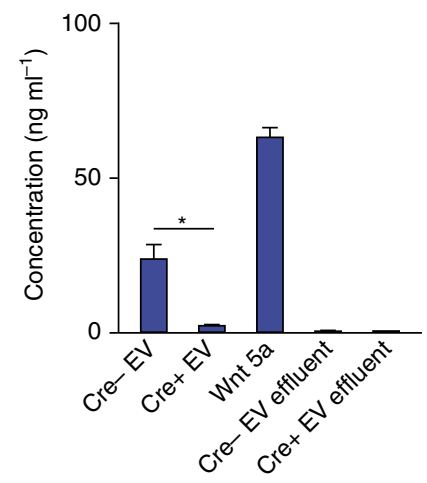

e

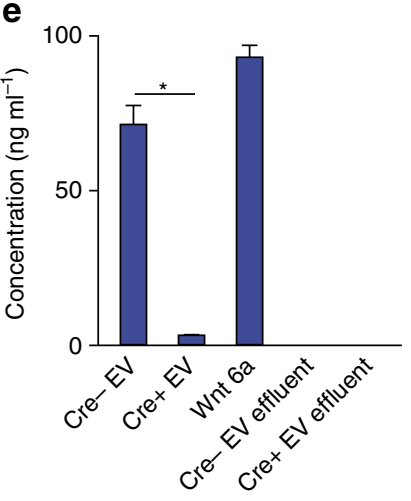

f

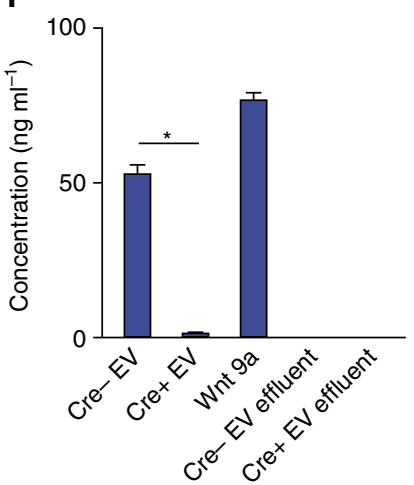

Figure 5 | Presence of EV-packaged WNT in BMMФ CM is critical for radio-mitigating function. (a) Kaplan-Meier survival analysis of WT mice ( $n=10$ per group) receiving CM/EV-depleted CM $(500 \mu \mathrm{l}$ per mice i.v.) derived from WT BMM $\Phi$ at $1 \mathrm{~h}$ and $48 \mathrm{~h}$ post $18.5 \mathrm{~Gy}$ AIR. Mice receiving WT BMM $\Phi$ CM showed $60 \%$ survival beyond 30 days compared with mice receiving EV-depleted WT BMMФ CM (using reagent $(P<0.004)$ or conventional method $(P<0.002)$ ) or untreated mice $(P<0.0009$; Log-rank (Mantel-Cox) test) where $80-100 \%$ of mice died within 12 days after irradiation ( $n=10$ per group). Reagent $(500 \mu \mathrm{l}$ per mice i.v.) used for chemical depletion of exosome did not confer any toxicity to normal mice. (b) Immunoblot to detect exosomal markers TSG101, ALIX and CD9 in EV from WT BMMФ CM or Porcn-null BMMФ CM or respective effluents. EV from WT BMMФ CM and from Porcn-null BMMФ CM showed the presence of EV markers. However, EV markers were not detected in effluents. (c) TCF/LEF reporter assay. HEK293 cells having TCF/LEF luciferase reporter construct were treated with EV (prepared with the conventional method) from WT or Porcn-null BMM Treatment with EV $\left(100 \mu \mathrm{g} \mathrm{ml}^{-1}\right)$ from WT BMMФ CM showed higher Luciferase activity compared with EV $\left(100 \mu \mathrm{g} \mathrm{ml}{ }^{-1}\right)$ from Porcn-null BMMФ CM $(P<0.0002)$ and effluent from WT BMMФ CM or Porcn-null BMMФ CM $(P<0.0001$ and $P<0.0002$ respectively; unpaired $t$-test, two-tailed). (d-f) ELISA to detect WNT5a, 6 and 9a in EVs from WT or Porcn-null BMMФ CM and effluents. Presence of WNT5a, WNT6 and WNT9a were detected in EVs from WT ВММФ CM (Cre - EV) but not in EVs from Porcn-null BMMФ CM (Cre + EV; $P<0.0002 ; P<2.78 \mathrm{E}-05$ and $P<3.26 \mathrm{E}-06$ respectively; unpaired $t$-test, two-tailed). WNT5a, WNT6 and WNT9a were also absent in effluents derived from WT or Porcn-null BMMФ CM. Recombinant WNT5a, WNT6 and WNT9a were used as positive control respectively.

that these WNTs were packaged in EVs (Fig. 5d-f). Therefore to confirm the radioprotective effect of these EVs, irradiated organoids were treated with EVs purified from WT or Porcnnull ВMМФ CM. Treatment with WT ВMМФ CM-derived EVs rescued organoids from radiation toxicity and improved organoid growth compared with irradiated control. However, treatment with Porcn-null BMMФ CM-derived EV did not show any radioprotective activity and failed to rescue organid growth (Fig. 7h). These data indicate that the canonical WNTs 5a, 6 and 9a are expressed by BMMФ in EVs and are effective in restoring crypt growth with the highest expressed Wnt 6 being the most efficacious. 
a

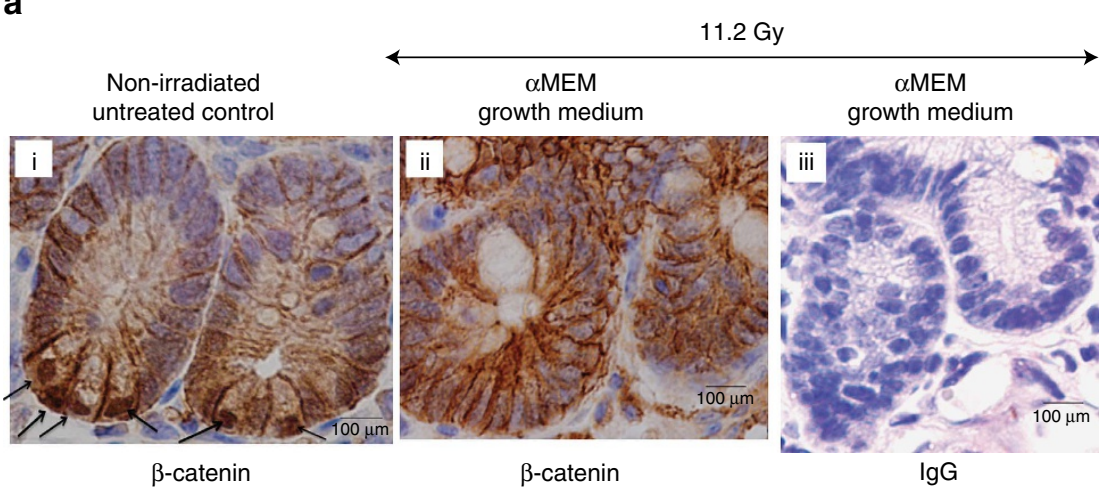

Csf1r.iCre; Porch ${ }^{\text {fl/t }}$

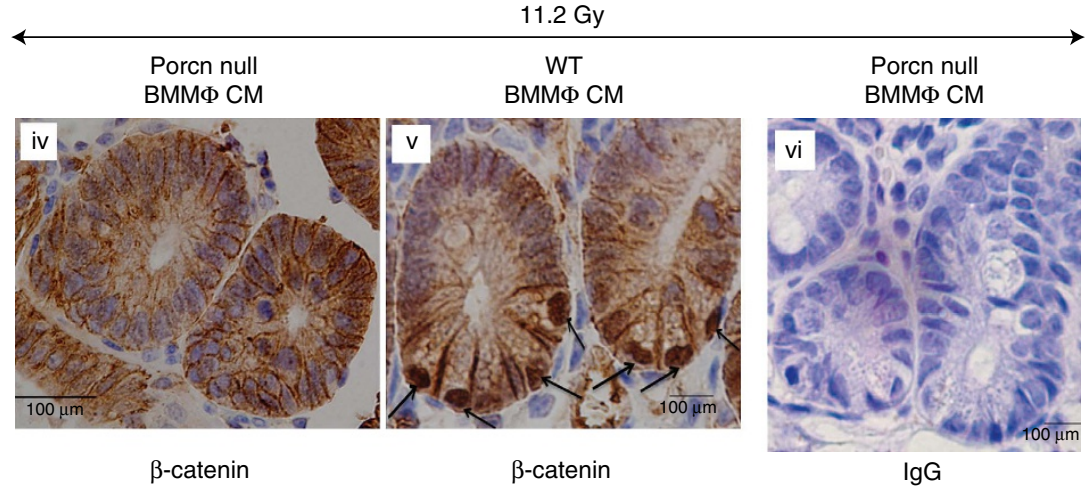

Csf1r.iCre; Porcn fl/ti

b

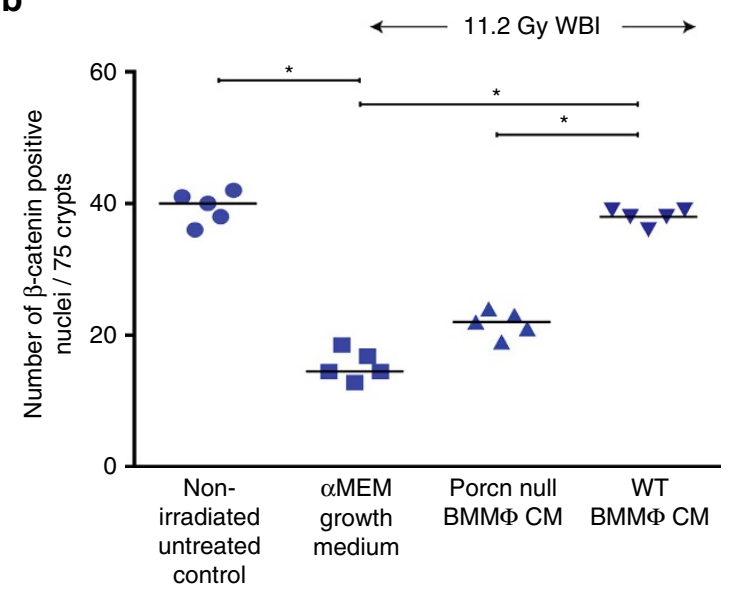

Figure 6 | Macrophage-derived WNTs induce $\boldsymbol{\beta}$-catenin activity in irradiated crypts. (a) Representative microscopic images ( $\times 60$ magnification) of jejunal sections immunostained with anti $\beta$-catenin antibody to determine $\beta$-catenin nuclear localization in CsflriCre;Porch ${ }^{f l / f l}$ mice. Nucleus stained with haematoxylin. Irradiated Csfir.iCre;Porcn ${ }^{f l / f l}$ mice receiving WT BMМФ CM (i.v.) showed more nuclear $\beta$-catenin staining (dark brown; indicated with arrows) at the base of the crypt compared with mice receiving Porcn-null BMMФ CM (i.v.) or $\alpha \mathrm{MEM}$ growth medium (ii; nucleus stained blue). Fig iii and vi are representative $\lg$ controls indicating lack of staining and thus showing specificity for the anti $\beta$-catenin antibody. (b) Nuclear $\beta$-catenin count: each data point is the average of the number of $\beta$-catenin-positive nucleus from 15 crypts per field, 5 fields per mice. Number of $\beta$-catenin-positive nucleus in irradiated Csf1riCre; Porcn ${ }^{f l / f l}$ mice receiving WT BMMФ CM is higher compared with Porcn-null BMMФ CM ( $\left.{ }^{\star} P<1 \mathrm{E}-04\right)$ or $\alpha M E M$ growth medium $\left({ }^{\star} P<1 \mathrm{E}-04\right)$. Treatment with Porcn-null BMMФ CM and $\alpha M E M$ growth medium following irradiation showed significantly fewer $\beta$-catenin-positive nuclei than the non-irradiated control ( ${ }^{\star} P<1.2 \mathrm{E}-04$ and ${ }^{\star} P<1 \mathrm{E}-04$ respectively; unpaired $t$-test, two-tailed).

\section{Discussion}

Rapid turnover of ISCs makes the intestinal mucosa especially vulnerable to high radiation exposure during radiotherapy. Therefore the gastrointestinal system is an early response organ to radiation. Consequently restoration of intestinal homoeostasis is critical to combat against RIGS. The present study indicates that macrophage-derived WNTs packaged in EVs are required for ISC self-renewal, proliferation and intestinal homoeostasis in response to radiation injury. To establish this involvement of macrophage-derived WNT in repair and regeneration of the intestine we used a mouse model where the gene expressing Porcn is selectively deleted in macrophages resulting in the loss of 
Table 1 | Real-time PCR analysis to determine mRNA levels of different $\beta$-catenin target genes in crypt epithelial cells.

\begin{tabular}{|c|c|}
\hline $\begin{array}{l}\text { Wnt target } \\
\text { genes }\end{array}$ & 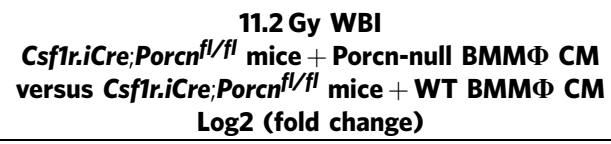 \\
\hline Ascl2 & $9.60 \pm 1.4$ \\
\hline Lgr5 & $4.44 \pm 0.9$ \\
\hline Plaur & $4.05 \pm 0.75$ \\
\hline Mmp9 & $3.82 \pm 0.82$ \\
\hline Ctgf & $3.08 \pm 1.1$ \\
\hline cMyc & $2.66 \pm 0.62$ \\
\hline Ptgs2 & $2.52 \pm 0.86$ \\
\hline Twist1 & $2.49 \pm 0.23$ \\
\hline Axin2 & $2.30 \pm 0.12$ \\
\hline Lef1 & $2.21 \pm 0.08$ \\
\hline Fosl1 & $2.04 \pm 0.02$ \\
\hline Pou5f1 & $2.01 \pm 0.03$ \\
\hline
\end{tabular}

release of WNT ligands. This deletion of Porcn in macrophages radio-sensitizes the mice to radiation doses that result in RIGS. Thus these mice die within 10-12 days post exposure and exhibit a disrupted crypt villus architecture compared with WT littermates. In addition we show that macrophage CM containing WNTs but not CM lacking WNTs can rescue the RIGS and result in survival of mice lacking Porcn in their macrophages. This rescue, however, is ablated if EVs are removed from the CM. These data can be replicated in intestinal organoid cultures designed to examine the role of LGR5 ${ }^{+ \text {ve }}$ ISCs in stem cell regeneration. This enabled us to identify canonical WNTs 5a, 6 and $9 \mathrm{a}$ that are expressed by macrophages able to induce regeneration. These data are consistent with the effects of BMMФ $\mathrm{CM}$ in inducing $\beta$-catenin nuclear localization in cryptal epithelial cells in vivo. Lethal irradiation of humans results in death due to RIGS and this pathology also limits radiotherapy for abdominal and pelvic cancers. Identification here that macrophage-derived EV-packaged WNTs can rescue this pathology with resultant survival of mice suggests that this might be an effective therapy for mitigation of RIGS following nuclear accidents as well as to increase the efficacy of radiotherapy.

Crypts present within the mouse small intestine have two types of stem cells. Bmil positive ISCs that are long-lived, label-retaining stem cells present at the +4 position of the crypt base. These $\mathrm{Bmil}^{+\mathrm{ve}}$ ISCs interconvert with more rapidly proliferating $\mathrm{LRG} 5^{+ \text {ve }}$ stem cells known as CBCs that express markers including Lgr5, Olfm4, Lrig1 and Ascl2.

These CBCs are also active stem cells, in as much as they are primarily involved in self-renewal and differentiation ${ }^{13,42-49}$. Although several reports suggested that intestinal stroma is the major source of WNT in the intestine ${ }^{17,50}$ the involvement of stromal WNTs in intestinal homoeostasis is not clear. Different cell types of intestinal stroma including endothelial cells, macrophages, neurons, fibroblasts and myofibroblasts could produce a cocktail of redundant WNT ligands maintaining intestinal homoeostasis in vivo. However, inhibition of WNT release from intestinal myofibroblast ${ }^{50}$ or from intestinal epithelium $^{1}$ does not affect intestinal homoeostasis. Pharmacological depletion of PORCN affecting both stromal and epithelial WNT release has shown that WNTs are required for recovery from radiation while depletion of WNT secretion by ablation of Porc using the Villin-cre in the intestinal epithelium indicated that this epithelium was not the WNT source during recovery from radiation. The present study has demonstrated that macrophages are the critical source of WNT for ISC regeneration as ВMМФ CM deficient in WNT obtained from the Csf1r.iCre;Porch flfl mice failed to rescue $\operatorname{Lgr}^{+ \text {ve }}$ ISCs following radiation injury. Moreover, macrophage-derived WNTs were secreted and trafficked through EVs as EV-depleted BMMФ $\mathrm{CM}$ is unable to mitigate radiation toxicity. However, macrophage-derived WNTs are not required for maintenance of normal intestinal homoeostasis as there were no phenotypic changes observed in the crypt architecture between non-irradiated WT and Csflr.iCre;Porcn flff mice.

Interestingly, in other organs macrophage WNTs are also required following injury. For example, macrophage WNT7b is required for kidney epithelial repair ${ }^{28}$. In addition, macrophage WNT signalling is required for stem cell regeneration in liver following damage ${ }^{29}$. Thus there appears to be a developing paradigm that macrophages deliver these important developmental molecules in a spatial and temporal specific way in adult animals to re-capitulate developmental processes to effect repair. In our studies on radiation repair we showed these macrophage-synthesized WNTs were contained within the EV fraction. While it is possible that the WNTs are in a contaminating vesicular fraction of another type it is also creditable that they are packaged in exosomes. While this remains to be formally proven it maybe that macrophagederived EV containing WNTs are responsible in all these examples indicating a paradigm for macrophage-regulated tissue repair. However, in some cases this delivery of WNTs appears to enhance pathology for example in cancer where macrophages may detect tissue damage caused by tumour growth $^{51}$ and respond by WNT production such as WNT7b. This macrophage-derived Wnt7b has been shown to enhance the growth and metastasis of tumours ${ }^{52}$. Understanding the control and molecular basis of these WNT signalling pathways in adults might therefore be of therapeutic use both to enhance repair as in this case against radiation-induced injury or to inhibit growth as in cancer.

\section{Methods}

Animals. Porcupine floxed (Porcn) embryos (FVB female donor + ICR male donor) were kindly provided by Dr J. Rossant (Hospital for Sick Children Research Institute, Toronto, Canada ${ }^{21}$. Embryos were transferred into oviducts of FvB females and the resultant pups bred to homozygosity after first back-crossing to the transgenic cre-recombinase strain, FVB.Csf1r-icre males for 3 generations (N3) to generate $\mathrm{Tg}\left(\mathrm{Csf} 1 \mathrm{r}\right.$.iCre)Jwp.-Porcn $\left.{ }^{\text {tmros }}\right)$ mice. Thereafter mice were inter-bred and cre-deleted mice compared with littermate cre-negative controls referred to as WT. All genotyping of mice was performed by PCR using the following primers $\left(5^{\prime}-3^{\prime}\right)$ : PorcnRecF1: 5' -CTGTTAAACCAAGACATGACCTTCA-3' ${ }^{\prime}$; PorcnRecR1, 5' ${ }^{\prime}$-TAA CTAGGACGCTTTGGGATAGGAT-3'; and PorcnRecR3: 5' -GTTCTGCCTTCC TAACCCATATAAC-3' (ref. 21). Amplicon sizes for primer combination F1-R1 representing floxed allele is $248 \mathrm{bp}$ and primer combination F1-R3 representing the Porcn-deleted allele is $386 \mathrm{bp}$. For Cre-recombinase forward: $5^{\prime}$-CTCTGACAGA TGCCAGGACA-3'; and Cre reverse: $5^{\prime}$-TCTCTGCCCAGAGTCATCCT-3'. For QPCR of genomic DNA and mRNA: Porcn-qPCR-F $5^{\prime}$-GCTGTCTCCTGCCT ACTGTCCA-3' Porcn-qPCR-R 5'-TGCTTGCATGCTTCAGGTAAGA-3'. All procedures involving mice were performed in accordance with National Institutes of Health regulations concerning the care and use of experimental animals. Experimental procedures were approved by Institutional Animal Care and Use Committee of the Albert Einstein College of Medicine and University of Kansas Medical Center. Female mice (6-8 weeks old) for both Csf1r.iCre;Porcn fl/fl and WT genotype were used for all the experiments. Male (6-8 weeks old) Lgr5/GFP-IRESCre-ERT2 knock-in mouse were used for all the experiments.

Isolation of ВММф. Bone marrow was isolated from both femurs and tibias of adult females Porcn;Csf1r.Cre mice and their WT littermate controls with $>95 \%$ purity ${ }^{53}$. Tibias were flushed and bone marrow cells were seeded into $10 \mathrm{~cm}^{2}$ tissue culture plates and cultured in alpha MEM (Cellgro) containing 10\% (v/v) fetal bovine serum (FBS) and 1\% penicillin-streptomycin, supplemented with M-CSF $10^{4} \mathrm{U} \mathrm{ml}^{-1}$ for $24 \mathrm{~h}$. To generate fully differentiated monocyte-derived macrophages non-adherent cells were transferred to petri dishes and cultured for 6-7 days at $37^{\circ} \mathrm{C}$. Macrophage CM was collected and then concentrated (10-fold) with centrifugal filter units (Millipore, Billerica, MA) before use for in vivo treatment $(500 \mu \mathrm{l}$ per mice, i.v.). For in vitro organoid studies mature bone marrow macrophages were cultured for $48 \mathrm{~h}$ in serum deprived medium $(0.5 \% \mathrm{v} / \mathrm{v}$ FBS $)$ 
and then CM was concentrated (10-fold) with centrifugal filter units. For pharmacological inhibition of Porcupine WT BMM $\phi$ were treated with C59, Porcupine Inhibitor II (Calbiochem; $20 \mu \mathrm{M}$ ). After $2 \mathrm{~h}$ of incubation cell culture medium (complete alpha MEM) having C59 was withdrawn and replaced with new medium without C59. One hour after WT BMMф CM was collected and concentrated for TOPFLASH assay (below).
TCF/LEF (TOPFLASH) reporter assay. To determine the canonical WNT activity in BMMф CM HEK293 cells (Signosis, Santa Clara, CA) having TCF/LEF luciferase reporter construct were treated with WT BMM $\phi \mathrm{CM}$ or Porcn-null $\mathrm{BMM} \phi \mathrm{CM}$ or EV-depleted WT BMM $\phi \mathrm{CM}$ or C59-treated WT BMM $\phi \mathrm{CM}$. $\mathrm{LiCl}(10 \mathrm{mM})$ treatment was used as positive control for luciferase activity. Luciferase activity was determined $24 \mathrm{~h}$ after using Dual-Luciferase Reporter Assay a

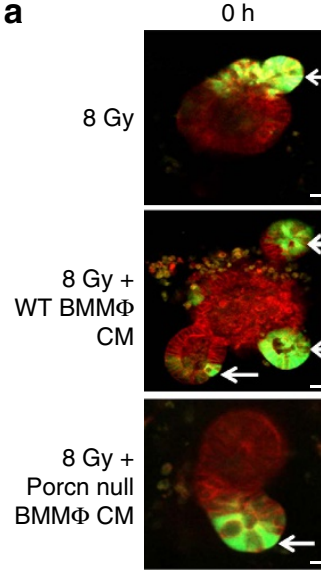

$24 \mathrm{~h}$
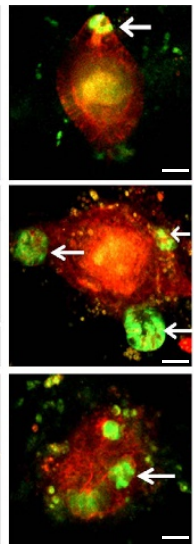

C

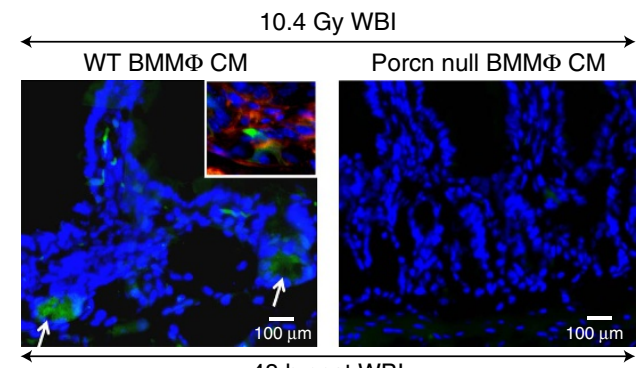

$48 \mathrm{~h}$ post WBI

e

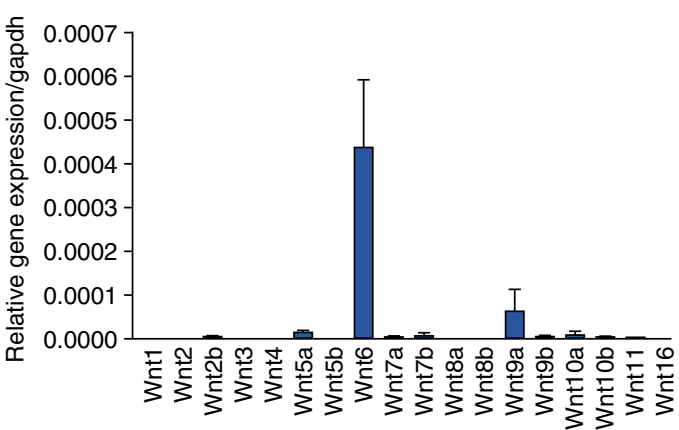

g

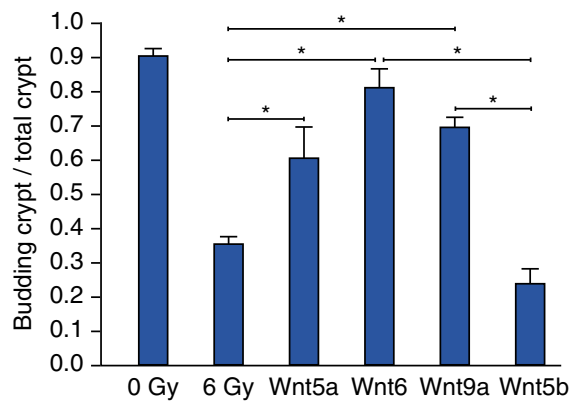

$48 \mathrm{~h}$
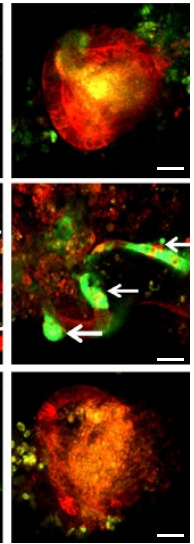

b

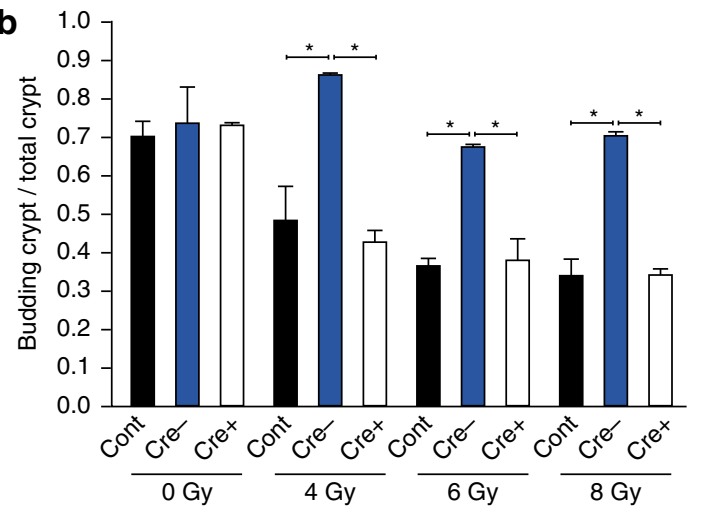

d

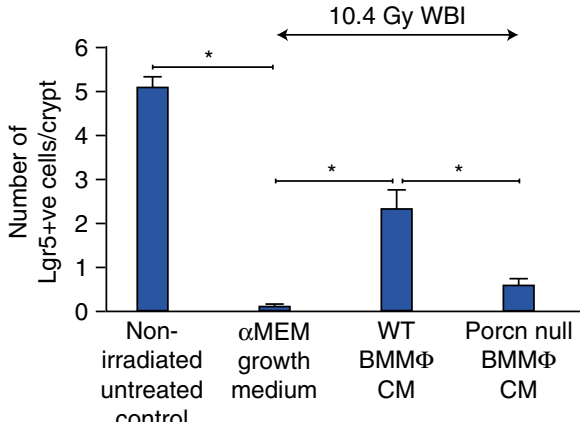

f

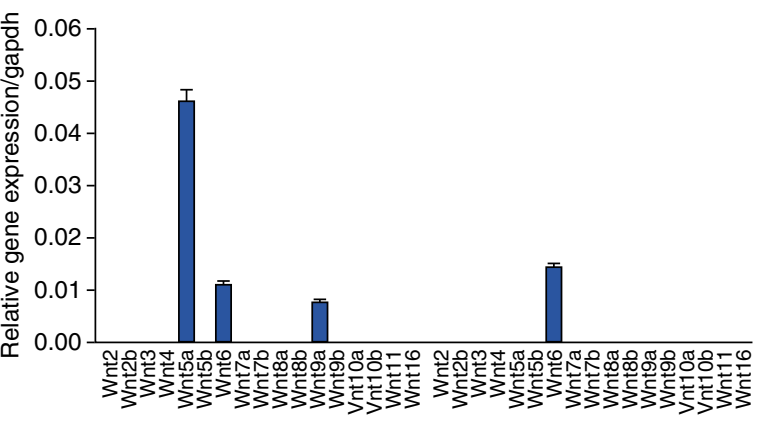

h

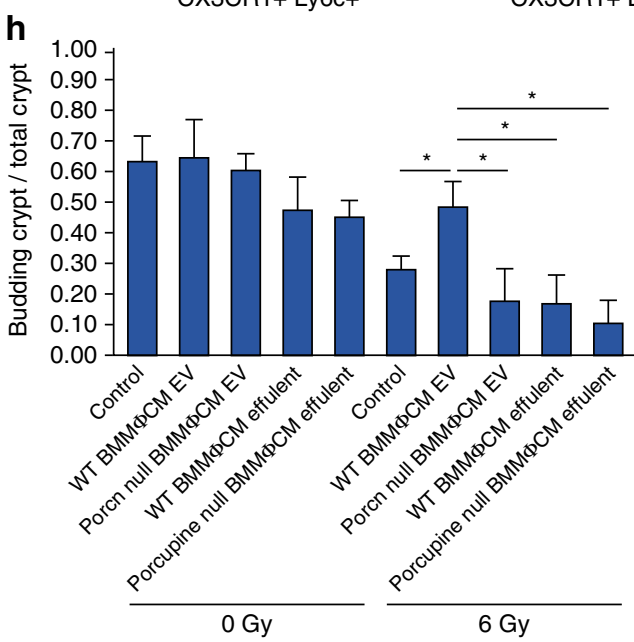


System (Promega) as per manufacturer's protocol. HEK293 cells having FOPFLASH construct (mutated TCF/LEF-binding site) were used as negative control. WNT activity was consistent between different batches of CM prepared under the same conditions.

HEK293 (human embryonic kidney) cell line was routinely characterized in the lab based on morphology and gene-expression patterns. Cells were confirmed to be free of mycoplasma contamination.

EV purification from WT BMM $\phi$ CM using EV Isolation Reagent. EV depletion in WT BMM $\phi$ CM was performed using Total Exosme Isolation Reagent (from cell culture media; Invitrogen) as per manufacturer's protocol. Effluent following EV purification was considered as depleted/EV-free fraction of WT BMM $\phi$ CM. Depletion of EV was confirmed by immunoblotting with exosome marker TSG101 (1:250; \#AB125011, Abcam, Cambridge, MA), ALIX (1:250; \#AB117600, Abcam) and CD9 (\#AB92726, Abcam). Absence of positive bands for all three exosomal markers in depleted fraction confirmed the EV depletion. However, positive bands for TSG101, ALIX and CD9 were detected in WT BMM $\phi$ CM and the purified EV fraction (All un-cropped western blots can be found in Supplementary Fig. 8). Average yield of EV was $60 \mu \mathrm{g}$ per $50 \mathrm{ml}$ of BMM $\phi \mathrm{CM}$. EV at $100 \mu \mathrm{g} \mathrm{ml}^{-1}$ was used for in vitro assays.

Purification of EV from BMM $\phi$ CM by conventional method. EVs were isolated from the WT/Porcn-null BMM $\phi$ CM as previously described ${ }^{54}$. Briefly, ВMМФ $\mathrm{CM}$ were centrifuged at $2,000 \mathrm{~g}$ for $20 \mathrm{~min}$ and $16,500 \mathrm{~g}$ for $30 \mathrm{~min}$ to remove other types of vesicles, such as microvesicles. The resulting cell-free medium was subjected to ultra-centrifugation at $100,000 \mathrm{~g}$ for $1 \mathrm{~h}$ to generate an EV pellet that was washed once with phosphate-buffered saline (PBS). The amount of EV protein recovered was assessed using detergent-compatible protein assay (Bio-Rad) according to the manufacturer's instructions. Average yield of EV was $75 \mu \mathrm{g}$ per $50 \mathrm{ml}$ of BMM $\phi$ CM. Purified EV at the concentration of $100 \mu \mathrm{g} \mathrm{ml}^{-1}$ and $200 \mathrm{\mu g} \mathrm{ml}^{-1}$ was used for TOPFLASH assay and ex vivo organoid assay, respectively.

ELISA of EV-packaged WNT. EV-packaged WNT was detected using ELISA starter accessory Kit (E101, Bethyl Laboratories, Montgomery, Texas) according to manufacturer's protocol. Biotin-conjugated antibodies against WNT5A, WNT9A (Bioss antibodies, Woburn, MA) and WNT6 (Novus Biologicals, Littleton, CO) was used for the ELISA. Purified EV with the concentration of $100 \mu \mathrm{g} \mathrm{ml}^{-1}$ was used for each assay.

Irradiation Procedure. WBI was performed on anaesthetised mice (intraperitoneal ketamine and xylazine $7: 1 \mathrm{mg} \mathrm{ml}^{-1}$ for $100 \mu \mathrm{l}$ per mouse) using a Mark I-68 A Cs-137 irradiator (JL Shepherd and Associates, San Fernando, CA) at

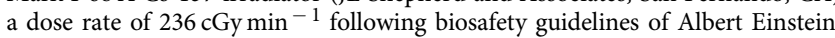
College of Medicine.

AIR was performed on anaesthetised mice (with a continuous flow $1.51 \mathrm{~min}^{-1}$ of $1.5 \%$ isoflurane in pure oxygen) using the small animal radiation research platform (SARRP, XStrahl, Surrey, UK). A $2 \mathrm{~cm}$ area of the mice containing the GI was irradiated (Fig. 2), thus shielding the upper thorax, head and neck as well as lower and upper extremities, protecting a significant portion of the bone marrow, thus inducing predominantly RIGS. A radiation dose of $18 \mathrm{~Gy}$ was delivered to the midline of the GI, ensuring homogeneous delivery by performing half of the total irradiation from the anterior-posterior direction and the second half from the posterior-anterior direction. The total irradiation time to deliver the intended dose was calculated with respect to dose rate, radiation field size and fractional depth dose to ensure accurate radiation dosimetry.

Histology. Since radiation doses $>8 \mathrm{~Gy}$ induces cell cycle arrest and apoptosis of the crypt epithelial cells within day 1 post-radiation, resulting in a decrease in regenerating crypt colonies by day 3.5 and ultimately villi denudation by day 7 post-radiation exposure ${ }^{2}$, we killed animals when moribund or at 3.5 and 7 days after WBI or AIR for time course experiments and intestines were collected for histology. The intestine of each animal was dissected, washed in PBS to remove intestinal contents and the jejunum was fixed in $10 \%$ neutral-buffered formalin before paraffin embedding. Tissue was routinely processed and cut into $5 \mu \mathrm{m}$ sections for haematoxylin and eosin and immunohistochemical staining. All haemotoxylin and eosin (HE) (Fisher Scientific, Pittsburgh, PA) staining was performed at the Histology and Comparative Pathology Facility in the Albert Einstein Cancer Center.

Crypt Proliferation Rate. To visualize villous cell proliferation, each mouse was injected intraperitoneally with $120 \mathrm{mg} \mathrm{kg}^{-1} \mathrm{BrdU}$ (Sigma-Aldrich, USA) 2 to $4 \mathrm{~h}$ before killing and mid-jejunum was collected for paraffin embedding and BrdU immunohistochemistry. Tissue sections were routinely deparaffinized and rehydrated through graded alcohols and incubated overnight at room temperature with a biotinylated monoclonal BrdU antibody (Zymed, South Francisco, CA). Nuclear staining was visualized using Streptavidin-peroxidase and diaminobenzidine (DAB) and samples were lightly counterstained with haematoxylin. Jejunum from mice, not injected with BrdU, was used as a negative control. Murine crypts were identified histologically according to the criteria established by Potten et al. ${ }^{55}$ Digital photographs of crypts were taken at high $(\times 40-60)$ magnification (Zeiss AxioHOME microscope) and crypt epithelial cells in intestinal sections were examined using Image software and classified as BrdU positive if they grossly demonstrated brown-stained nuclei from DAB staining or as BrdU negative if they were blue stained nuclei. The proliferation rate was calculated as the percentage of BrdU-positive cells over the total number of cells in each crypt. A total of 60 crypts were examined per animal.

Determination of Villi Length and Crypt Depth. Crypt depth was independently and objectively analysed and quantitated in a blind manner from coded digital photographs of crypts from HE-stained slides using Image 1.37 software to measure the height in pixels from the bottom of the crypt to the crypt villus junction. Villi length was determined by measuring the length from the crypt villus junction to villous tip. This measurement in pixels was converted to length (in $\mu \mathrm{m}$ ) by dividing with the following a conversion factor $\left(1.46\right.$ pixels $\left.\mu \mathrm{m}^{-1}\right)$.

$\beta$-catenin immunohistochemistry of mouse jejunum. $\beta$-Catenin immunohistochemistry was performed in paraffin-embedded sections of mouse jejunum ${ }^{56}$ Before immunostaining antigen retrieval was performed by heating slides in $\mathrm{pH} 6.0$ citrate buffer at $100{ }^{\circ} \mathrm{C}$ for $20 \mathrm{~min}$ in a microwave oven at $500 \mathrm{~W}$ using antigen retrieval solution (10 mM Tris and $1 \mathrm{mM}$ EDTA, $\mathrm{pH} 9.0$ ). Non-specific antibody binding was blocked for $20 \mathrm{~min}$ by incubation with $0.05 \% \mathrm{w} / \mathrm{v}$ BSA in PBS. Tissue was stained using the anti- $\beta$-catenin Antibody (1:100 dilution; BD Transduction Laboratories, Franklin Lakes, NJ; \#610154) at room temperature for $2 \mathrm{~h}$ followed by staining with horseradish peroxidase-conjugated Anti-Mouse Antibody (Dako, Denmark) at room temperature for $1 \mathrm{~h}$. Peroxidase activity was detected by adding

Figure 7 | CM from WT ВMМФ rescued the Lgr5 ${ }^{+ \text {ve }}$ ISC population in both in vivo and in vitro. (a) Two-photon microscopic images of organoids from Lgr5/GFP-IRES-Cre-ERT2 knock-in mouse irradiated and then treated with CM. CM from WT BMMФ (Cre-) rescued the LGR5+ve (GFP+ve cells) ISC population from radiation injury as indicated by arrow. GFP + ve cells disappeared in organoids treated with/without Porcn-null BMM $\mathrm{B} C M(\mathrm{Cre}+)$ within $48 \mathrm{~h}$ of exposure to $8 \mathrm{~Gy}$ irradiation. Scale bar $=50 \mu \mathrm{M}$. (b) Histograms demonstrating the effect of Cre $\pm \mathrm{CM}$ treatment on crypt organoid growth following irradiation. Treatment groups: $4 \mathrm{~Gy}$ versus $4 \mathrm{~Gy}+\mathrm{Cre}-\mathrm{CM}\left({ }^{\star} P<0.013\right), 6 \mathrm{~Gy}$ versus $6 \mathrm{~Gy}+\mathrm{Cre}-\mathrm{CM}\left({ }^{\star} P<0.001\right), 8 \mathrm{~Gy}$ versus

$8 \mathrm{~Gy}+\mathrm{Cre}-\mathrm{CM}\left({ }^{\star} P<0.004\right.$; unpaired $t$-test, two-tailed). (c) Representative images of jejunal sections demonstrating the presence of GFP + ve $L g r 5+v e$ ISCs (arrow) in Lgr5/GFP-IRES-Cre-ERT2 knock-in mice receiving WT BMMФ CM. Note, the absence of GFP+ve cells in mice receiving Porcn-null BMM $\Phi$ CM. Nuclei are stained with DAPI. Top panel Inset: phalloidin (red) staining to show localization of cell membrane. (d) Histograms demonstrating the number of GFP + ve Lgr5 +ve ISCs/crypt in jejunal sections from Lgr5/GFP-IRES-Cre-ERT2 knock-in mice exposed to irradiation and then treated with Porcnnull or WT ВММФ CM. Irradiated mice receiving WT BMMФ CM showed higher numbers of GFP + ve cells compared with mice receiving Porcn-null BMMФ CM $\left({ }^{\star} P<0.0002\right)$ or $\alpha M E M\left({ }^{\star} P<1.74858 \mathrm{E}-06\right.$; unpaired $t$-test, two-tailed). (e) qPCR analysis of RNA from WT BMMФ demonstrated mRNA expression of Wnt5a, Wnt6 and Wnt9a. (f) qPCR analysis of intestinal macrophages RNA from WT mice. (g) Organoids were exposed to irradiation (6 Gy) and treated with Porcn-null ВММФ CM supplemented with Wnt5a, Wnt6, Wnt9a and Wnt5b $\left(1 \mu \mathrm{g} \mathrm{ml}^{-1}\right)$. Organoid survival was improved with treatment of canonical WNT ligands WNT5a $\left({ }^{\star} P<0.009\right)$, WNT6 $\left({ }^{\star} P<0.0001\right)$ and WNT9a $\left({ }^{\star} P<8.99662 \mathrm{E}-05\right)$ compared with irradiated control. However, treatment with non-canonical WNT5b failed to rescue organoids from radiation lethality (WNT6 versus WNT5b * $P<0.0001$; WNT9a versus WNT5b ${ }^{\star} P<0.0001$; unpaired $t$-test, two-tailed). (h) Histogram demonstrating the effect of EVs from WT/Porcn-null BMMФ CM on crypt organoid growth following irradiation. Treatment groups: $6 \mathrm{~Gy}$ (control) versus $6 \mathrm{~Gy}+\mathrm{WT}$ BMMФ CM EV ( $\left.{ }^{\star} P<0.009\right), 6 \mathrm{~Gy}+$ WT BMMФ CM EV versus $6 \mathrm{~Gy}+$ Porcn-null BMMФ CM EV $\left({ }^{\star} P<0.004\right), 6 \mathrm{~Gy}+\mathrm{WT}$ BMM $\Phi \mathrm{CM}$ EV versus $6 \mathrm{~Gy}+\mathrm{WT}$ BMM $\Phi \mathrm{CM}$ effluent $\left({ }^{\star} P<0.0002\right), 6 \mathrm{~Gy}+\mathrm{WT}$ BMM $\Phi \mathrm{CM} \mathrm{EV}$ versus Porcn-null BMMФ CM effluent $\left({ }^{\star} P<0.0001\right.$; unpaired $t$-test, two-tailed). 
DAB substrate. Nucleus was counter-stained with haematoxylin (blue). $\beta$-Catenin-positive nucleus (stained dark brown) was calculated from 15 crypts per field, 5 fields per mice.

Isolation of Intestinal Epithelial Cells. Intestinal epithelial cells were prepared from the jejunum of adult male C57Bl6 mice ${ }^{57}$. Mice were anaesthetized and a catheter was inserted into the intestine through an incision in the most proximal part of duodenum. A second incision was made just proximal to the caecum and the entire small intestine was perfused with ice-cold PBS and then flushed twice with ice-cold PBS plus $1 \mathrm{mM}$ dithiothreitol (DTT). The duodenum and ileum were discarded and the entire jejunum was tied at the distal end and filled to distension with isolation citrate buffer $(0.9 \% \mathrm{w} / \mathrm{v} \mathrm{NaCl}, 1.5 \mathrm{mM} \mathrm{KCl}, 27.0 \mathrm{mM}$ Na Citrate, $8.0 \mathrm{mM} \mathrm{KH} 2 \mathrm{PO} 4$ and $5.6 \mathrm{mM} \mathrm{Na} 2 \mathrm{HPO} 4, \mathrm{pH} 7.3$ ) heated to $37^{\circ} \mathrm{C}$ for $15 \mathrm{mins}$. After incubation, the jejunum was emptied and filled with $5 \mathrm{ml}$ ethylene diamine tetra acetic acid (EDTA) buffer $(0.9 \% \mathrm{w} / \mathrm{v} \mathrm{NaCl}, 8 \mathrm{mM} \mathrm{KH} 2 \mathrm{PO} 4,5.6 \mathrm{mM}$ Na2HPO4, $1.5 \mathrm{mM} \mathrm{Na2-EDTA,} \mathrm{pH} \mathrm{7.6,} \mathrm{plus} \mathrm{0.5} \mathrm{mM} \mathrm{DTT} \mathrm{and} \mathrm{0.23} \mathrm{mM} \mathrm{PMSF;}$ Sigma-Aldrich, St Louis, MO). Each jejunum was then physically manipulated and tapped allowing the cells to separate from the interior surface. The jejunum was finally rinsed twice with $5 \mathrm{ml}$ of EDTA buffer and all the fluid containing epithelial cells was collected, centrifuged at $300 \mathrm{~g}$ for $5 \mathrm{~min}$, washed twice with $20 \mathrm{ml}$ of balanced salt solution (BSS) containing $135 \mathrm{mM} \mathrm{NaCl}, 4.5 \mathrm{mM} \mathrm{KCl}, 5.6 \mathrm{mM}$ glucose, $0.5 \mathrm{mM} \mathrm{MgCl} 2,10 \mathrm{mM}$ HEPES and $1.0 \mathrm{mM} \mathrm{CaCl} 2, \mathrm{pH} 7.4$, and the cells suspended in $2 \mathrm{ml}$ of the same solution. Cell numbers were determined with haemocytometer and viability (>90 $\pm 5 \%$ ) was assessed using trypan blue exclusion.

Isolation and flowcytometric sorting of intestinal $\mathbf{M \Phi . ~ I s o l a t i o n ~ o f ~ I n t e s t i n a l ~}$ lamina propria cells was performed by following a method established previously ${ }^{58}$ with slight modifications. Small intestines were washed with three times with HBSS $(\mathrm{Ca} / \mathrm{Mg}$-free), and fat and Peyer's patches were removed. Small intestines were then opened longitudinally, cut into $1-\mathrm{cm}$ pieces, and incubated in HBSS containing $5 \mathrm{uM}$ EDTA $+5 \%$ FBS $+1 \mu \mathrm{M}$ DTT. Tissue was then digested 0.14 Wünsch $\mathrm{U} \mathrm{ml}^{-1}$ Liberase (Sigma) for $30 \mathrm{mins}$ at $37^{\circ} \mathrm{C}$ on a rotor. The digested cell suspension was then passed through $100 \mu \mathrm{m}$ cell strainers. Isolated intestinal cells were stained with CD45 Percpcy5.5 (0.35 $\mu \mathrm{l}$ per $100 \mu \mathrm{l}$; \#45-0451-82, eBioscience, San Diego, CA) CX3CR1 PE-TexasRed ( $1.5 \mu \mathrm{l}$ per $100 \mu \mathrm{l}$; \#149013, Biolegend, San Diego, CA) F4/80 AF647 (8 $\mu \mathrm{l}$ per $100 \mu$ l; \#MCA497A647, Bio-Rad, Hercules, CA), CD11b eflour605 (2 $\mu \mathrm{l}$ per $100 \mu \mathrm{l}$; 83-0112-42, eBioscience) Ly6C PE/cy7 (1 $\mu \mathrm{l}$ per $100 \mu \mathrm{l}$; \#128018, Biolegend) Ly6G APC/cy7 (0.3 $\mu \mathrm{l}$ per $100 \mu \mathrm{l}$; \#127624, Biolegend) and were subjected to flowcytometric sorting to purify intestinal macrophages using FACS Aria machine (BD)

Real-time PCR to determine $\boldsymbol{\beta}$-catenin target genes mRNA level. To compare the mRNA levels of $\beta$-catenin target genes in intestinal crypt cells from irradiated mice treated with Porcn-null BMMФ CM or WT BMMФ CM real-time PCR were performed using real-time array system from Qiagen. RNA was isolated from crypt cells using RNeasy mini kit from Qiagen. Preparation of cDNA followed by real-time PCR array was performed according to manufacturer protocol.

FITC-dextran permeability assay. At day 5 post WBI mice were gavaged with $0.6 \mathrm{mg} \mathrm{g}^{-1}$ body weight of a FITC-dextran solution $(4,000 \mathrm{kD}$ size, Sigma). In all, $4 \mathrm{~h}$ after gavage mice were killed and serum was obtained with cardiac puncture ${ }^{59}$. Samples were measured in a 96-well plate using a Flexstation ii 384 multiwell fluorometer (Molecular Devices). A standard curve was constructed using mouse serum having increasing amounts of FITC-dextran to determine the serum levels of FITC-dextran in different treatment groups.

Preparation in vitro culture of intestinal crypt organoids. Small intestine from Lgr5-GFP mice, or their littermates control mice was used for Crypt isolation and development of ex vivo organoid culture ${ }^{60-62}$. The tissue was scraped for removing villi and chopped into $\sim 5 \mathrm{~mm}$ pieces. Then tissue was washed with cold PBS, and incubated in $2.5 \mathrm{mM}$ EDTA in PBS for $60 \mathrm{~min}$ on ice. The tissue fragments were suspended vigorously with a 10 -ml pipette in cold $10 \% \mathrm{v} / \mathrm{v}$ FBS, yielding supernatants enriched in crypts. Crypt fractions were centrifuged at $300 \mathrm{~g}$ for $5 \mathrm{~min}$ at $4{ }^{\circ} \mathrm{C}$ and diluted with advanced DMEM/F12 (Invitrogen) containing B27, N2, $1 \mu \mathrm{M} \mathrm{n}$-Acetylcysteine, $10 \mathrm{mM}$ HEPES, penicillin/streptomycin, and Glutamax (all Invitrogen). Samples were passed through $100 \mu \mathrm{m}$ filters (BD Biosciences), and centrifuged at $275 \mathrm{~g}$ for $5 \mathrm{~min}$ at $4^{\circ} \mathrm{C}$ and single cells were discarded. Crypts were embedded in extracellular matrix (provided from NCI) and seeded on pre-warmed 24-well plate. After the matrix solidified, advanced DMEM/F12 medium containing $50 \mathrm{ng} \mathrm{ml}^{-1} \mathrm{EGF}$ (Invitrogen), $100 \mathrm{ng} \mathrm{ml}^{-1}$ Noggin (Peprotech), $1 \mu \mathrm{g} \mathrm{ml}^{-1}$ RSPO1 was overlaid. Growth factors were added every other day and the entire medium was changed twice a week. Passage was performed at day 7 . The number of organoids per well was counted on microscopic images. The images of organoids were acquired using fluorescent microscopy (Nikon, TE2000-U) and two-photon microscopy (Nikon, A1RMP). For WNT supplementation experiments WNT5A, WNT9A (R\&D Systems) and WNT6 (Novus) was used with the concentration of $1 \mu \mathrm{g} \mathrm{ml}^{-1}$. Total number of crypt structures and number of budding crypts were counted and expressed as a ratio of budding crypts/total crypt structure.

Statistics. Mice survival/mortality in different treatment group was analysed by Kaplan-Meier statistics as a function of radiation dose using Graphpad Prism-6.0 software for Mac. Mice were sorted randomly after genotyping to each experimental and control group. Minimum number of mice used for survival/ mortality study was $n=10$ per group. For intestinal sampling regions were chosen at random for digital acquisition for quantitation. Digital image data was evaluated in a blinded manner as to treatment. A two-sided Student's $t$-test was used to determine significant differences between experimental cohorts $(P<0.05)$ with representative standard errors of the mean.

Study approval. All the animals were maintained in the animal maintenance facilities and all animal studies were performed under the guidelines and protocols of the Institutional Animal Care and Use Committee of the Albert Einstein College of Medicine and University of Kansas Medical Center.

Data availability. The authors declare that all data supporting the findings of this study are available within the article and its Supplementary Information files or from the corresponding author on reasonable request.

\section{References}

1. Kabiri, Z. et al. Stroma provides an intestinal stem cell niche in the absence of epithelial Wnts. Development 141, 2206-2215 (2014).

2. Saha, S. et al. Bone marrow stromal cell transplantation mitigates radiationinduced gastrointestinal syndrome in mice. PLoS ONE 6, e24072 (2011).

3. Smith, P. D. et al. Intestinal macrophages and response to microbial encroachment. Mucosal. Immunol. 4, 31-42 (2011).

4. Pull, S. L., Doherty, J. M., Mills, J. C., Gordon, J. I. \& Stappenbeck, T. S. Activated macrophages are an adaptive element of the colonic epithelial progenitor niche necessary for regenerative responses to injury. Proc. Natl Acad. Sci. USA 102, 99-104 (2005).

5. Saha, S. et al. TLR9 agonist protects mice from radiation-induced gastrointestinal syndrome. PLoS ONE 7, e29357 (2012).

6. Kuhnert, F. et al. Essential requirement for Wnt signaling in proliferation of adult small intestine and colon revealed by adenoviral expression of Dickkopf-1. Proc. Natl Acad. Sci. USA 101, 266-271 (2004).

7. Gregorieff, A. et al. Expression pattern of Wnt signaling components in the adult intestine. Gastroenterology 129, 626-638 (2005).

8. Clevers, H. \& Nusse, R. Wnt/beta-catenin signaling and disease. Cell 149, 1192-1205 (2012).

9. Bhanja, P. et al. Protective role of R-spondin1, an intestinal stem cell growth factor, against radiation-induced gastrointestinal syndrome in mice. PLOS ONE 4, e8014 (2009).

10. Kim, K. A. et al. Mitogenic influence of human R-spondin1 on the intestinal epithelium. Science 309, 1256-1259 (2005).

11. Zhao, J. et al. R-Spondin 1 protects mice from chemotherapy or radiationinduced oral mucositis through the canonical Wnt/beta-catenin pathway. Proc. Natl Acad. Sci. USA 106, 2331-2336 (2009)

12. Zhao, J. et al. R-spondin1, a novel intestinotrophic mitogen, ameliorates experimental colitis in mice. Gastroenterology 132, 1331-1343 (2007)

13. Yan, K. S. et al. The intestinal stem cell markers Bmil and Lgr5 identify two functionally distinct populations. Proc. Natl Acad. Sci. USA 109, 466-471 (2012).

14. Cervantes, S., Yamaguchi, T. P. \& Hebrok, M. Wnt5a is essential for intestinal elongation in mice. Dev. Biol. 326, 285-294 (2009).

15. Davies, P. S., Dismuke, A. D., Powell, A. E., Carroll, K. H. \& Wong, M. H. Wnt-reporter expression pattern in the mouse intestine during homeostasis. BMC. Gastroenterol. 8, 57 (2008).

16. Zeve, D. et al. Wnt signaling activation in adipose progenitors promotes insulin-independent muscle glucose uptake. Cell. Metab. 15, $492-504$ (2012).

17. Farin, H. F., Van Es, J. H. \& Clevers, H. Redundant sources of Wnt regulate intestinal stem cells and promote formation of Paneth cells. Gastroenterology 143, 1518-1529 e1517 (2012).

18. Najdi, R. et al. A uniform human Wnt expression library reveals a shared secretory pathway and unique signaling activities. Differentiation. 84, 203-213 (2012).

19. Proffitt, K. D. \& Virshup, D. M. Precise regulation of porcupine activity is required for physiological Wnt signaling. J. Biol. Chem. 287, 34167-34178 (2012).

20. Hofmann, K. A superfamily of membrane-bound O-acyltransferases with implications for wnt signaling. Trends. Biochem. Sci. 25, 111-112 (2000).

21. Biechele, S., Cox, B. J. \& Rossant, J. Porcupine homolog is required for canonical Wnt signaling and gastrulation in mouse embryos. Dev. Biol. 355, 275-285 (2011). 
22. Kadowaki, T., Wilder, E., Klingensmith, J., Zachary, K. \& Perrimon, N. The segment polarity gene porcupine encodes a putative multitransmembrane protein involved in Wingless processing. Genes Dev. 10, 3116-3128 (1996).

23. Tanaka, K., Okabayashi, K., Asashima, M., Perrimon, N. \& Kadowaki, T. The evolutionarily conserved porcupine gene family is involved in the processing of the Wnt family. Eur. J. Biochem. 267, 4300-4311 (2000).

24. Coombs, G. S. et al. Modulation of Wnt/beta-catenin signaling and proliferation by a ferrous iron chelator with therapeutic efficacy in genetically engineered mouse models of cancer. Oncogene 31, 213-225 (2012).

25. Janda, C. Y., Waghray, D., Levin, A. M., Thomas, C. \& Garcia, K. C. Structural basis of Wnt recognition by Frizzled. Science 337, 59-64 (2012).

26. Stefater, 3rd J. A., Ren, S., Lang, R. A. \& Duffield, J. S. Metchnikoff s policemen: macrophages in development, homeostasis and regeneration. Trends. Mol. Med. 17, 743-752 (2011)

27. Wynn, T. A., Chawla, A. \& Pollard, J. W. Macrophage biology in development, homeostasis and disease. Nature 496, 445-455 (2013).

28. Lin, S. L. et al. Macrophage Wnt7b is critical for kidney repair and regeneration. Proc. Natl Acad. Sci. USA 107, 4194-4199 (2010).

29. Boulter, L. et al. Macrophage-derived Wnt opposes Notch signaling to specify hepatic progenitor cell fate in chronic liver disease. Nat. Med. 18, 572-579 (2012).

30. Deng, L. et al. A novel mouse model of inflammatory bowel disease links mammalian target of rapamycin-dependent hyperproliferation of colonic epithelium to inflammation-associated tumorigenesis. Am. J. Pathol. 176, 952-967 (2010).

31. Raposo, G. \& Stoorvogel, W. Extracellular vesicles: exosomes, microvesicles, and friends. J. Cell Biol. 200, 373-383 (2013).

32. Korinek, V. et al. Constitutive transcriptional activation by a beta-catenin-Tcf complex in APC-/- colon carcinoma. Science 275, 1784-1787 (1997).

33. Jackson, Jr. W. L., Gallagher, C., Myhand, R. C. \& Waselenko, J. K. Medical management of patients with multiple organ dysfunction arising from acute radiation syndrome. BJR Suppl. 27, 161-168 (2005).

34. Leibowitz, B. J. et al. Ionizing irradiation induces acute haematopoietic syndrome and gastrointestinal syndrome independently in mice. Nat. Commun. 5, 3494 (2014).

35. Dawson, P. A. et al. Reduced mucin sulfonation and impaired intestinal barrier function in the hyposulfataemic NaS1 null mouse. Gut 58, 910-919 (2009).

36. Zhang, L. \& Wrana, J. L. The emerging role of exosomes in Wnt secretion and transport. Curr. Opin. Genet. Dev. 27, 14-19 (2014).

37. Gross, J. C., Chaudhary, V., Bartscherer, K. \& Boutros, M. Active Wnt proteins are secreted on exosomes. Nat. Cell. Biol. 14, 1036-1045 (2012).

38. Takada, R. et al. Monounsaturated fatty acid modification of Wnt protein: its role in Wnt secretion. Dev. Cell. 11, 791-801 (2006).

39. Willert, K. et al. Wnt proteins are lipid-modified and can act as stem cell growth factors. Nature 423, 448-452 (2003).

40. Mikels, A. J. \& Nusse, R. Purified Wnt5a protein activates or inhibits beta-catenin-TCF signaling depending on receptor context. PLoS Biol. 4, e115 (2006).

41. Tanaka, S., Terada, K. \& Nohno, T. Canonical Wnt signaling is involved in switching from cell proliferation to myogenic differentiation of mouse myoblast cells. J. Mol. Signal. 6, 12 (2011)

42. Barker, N., van de Wetering, M. \& Clevers, H. The intestinal stem cell. Genes Dev. 22, 1856-1864 (2008)

43. Buczacki, S. J. et al. Intestinal label-retaining cells are secretory precursors expressing Lgr5. Nature 495, 65-69 (2013).

44. Powell, D. W. \& Saada, J. I. Mesenchymal stem cells and prostaglandins may be critical for intestinal wound repair. Gastroenterology 143, 19-22 (2012).

45. Takeda, N. et al. Interconversion between intestinal stem cell populations in distinct niches. Science 334, 1420-1424 (2011).

46. van der Flier, L. G., Haegebarth, A., Stange, D. E., van de Wetering, M. \& Clevers, H. OLFM4 is a robust marker for stem cells in human intestine and marks a subset of colorectal cancer cells. Gastroenterology 137, 15-17 (2009).

47. Wong, V. W. et al. Lrig1 controls intestinal stem-cell homeostasis by negative regulation of ErbB signalling. Nat. Cell. Biol. 14, 401-408 (2012).

48. Hayakawa, Y. et al. CCK2R identifies and regulates gastric antral stem cell states and carcinogenesis. Gut 64, 544-553 (2014).

49. Metcalfe, C., Kljavin, N. M., Ybarra, R. \& de Sauvage, F. J. Lgr5 + stem cells are indispensable for radiation-induced intestinal regeneration. Cell Stem Cell 14, 149-159 (2014)
50. San Roman, A. K., Jayewickreme, C. D., Murtaugh, L. C. \& Shivdasani, R. A. Wnt secretion from epithelial cells and subepithelial myofibroblasts is not required in the mouse intestinal stem cell niche in vivo. Stem Cell Rep. 2, 127-134 (2014).

51. Pollard, J. W. Tumour-educated macrophages promote tumour progression and metastasis. Nat. Rev. Cancer 4, 71-78 (2004).

52. Yeo, E. J. et al. Myeloid WNT7b mediates the angiogenic switch and metastasis in breast cancer. Cancer Res. 74, 2962-2973 (2014).

53. Stanley, E. R. Murine bone marrow-derived macrophages. Methods Mol. Biol. 5 , 299-302 (1990).

54. Atay, S. et al. Oncogenic KIT-containing exosomes increase gastrointestinal stromal tumor cell invasion. Proc. Natl Acad. Sci. USA 111, 711-716 (2014).

55. Potten, C. S., Booth, C. \& Pritchard, D. M. The intestinal epithelial stem cell: the mucosal governor. Int. J. Exp. Pathol. 78, 219-243 (1997).

56. Barker, N. \& van den Born, M. Detection of beta-catenin localization by immunohistochemistry. Methods Mol. Biol. 468, 91-98 (2008).

57. Ferraris, R. P. \& Diamond, J. Crypt-villus site of glucose transporter induction by dietary carbohydrate in mouse intestine. Am. J. Physiol. 262, G1069-G1073 (1992).

58. Goodyear, A. W., Kumar, A., Dow, S. \& Ryan, E. P. Optimization of murine small intestine leukocyte isolation for global immune phenotype analysis. J. Immunol. Methods 405, 97-108 (2014).

59. Karhausen, J. et al. Epithelial hypoxia-inducible factor-1 is protective in murine experimental colitis. J. Clin. Invest. 114, 1098-1106 (2004).

60. Sato, T. et al. Paneth cells constitute the niche for Lgr5 stem cells in intestinal crypts. Nature 469, 415-418 (2011).

61. Sato, T. et al. Single Lgr5 stem cells build crypt-villus structures in vitro without a mesenchymal niche. Nature 459, 262-265 (2009).

62. Barker, N. et al. Lgr5 ( + ve) stem cells drive self-renewal in the stomach and build long-lived gastric units in vitro. Cell Stem Cell 6, 25-36 (2010).

\section{Acknowledgements}

This work was supported by grants from the Wellcome Trust 101067/Z/13/Z (J.W.P.), Medical Research Council Centre for Reproductive Health grant G1002033 (J.W.P.) Paul Calabresi Career Development Award 5K12CA132783 (S.S.), K01DK096032 (S.S.), ACS IRG Pilot (S.S.), KUCC support grant (S.S.), 1 U19 AI091175 (C.G.) and 1U01 DK103155 (T.C.W. and C.G.). We thank Drs Bichele and Rossant, Hospital for Sick Children, Toronto, Canada for the mice carrying the floxed allele of Porcn and advice on genotyping.

\section{Author contributions}

Conceived and designed the experiments: S.S., E.A., T.C.W., C.G. and J.W.P. Performed the experiments: S.S., E.A., Y.H., P.B., N.P.B., W.A.T., Y.T., S.A. and L.L. Analysed the data: S.S., E.A., Y.H., P.B. and J.W.P. Contributed reagents/materials/analysis tools: S.S., T.C.W., C.G., A.K.G. and J.W.P. Wrote the paper: S.S., E.A. and J.W.P.

\section{Additional information}

Supplementary Information accompanies this paper at http://www.nature.com/ naturecommunications

Competing financial interests: The authors declare no competing financial interests.

Reprints and permission information is available online at http://npg.nature.com/ reprintsandpermissions/

How to cite this article: Saha, S. et al. Macrophage-derived Extracellular Vesicle-packaged WNTs rescue intestinal stem cells and enhance survival after radiation injury. Nat. Commun. 7, 13096 doi: 10.1038/ncomms13096 (2016).

This work is licensed under a Creative Commons Attribution 4.0 International License. The images or other third party material in this article are included in the article's Creative Commons license, unless indicated otherwise in the credit line; if the material is not included under the Creative Commons license, users will need to obtain permission from the license holder to reproduce the material. To view a copy of this license, visit http://creativecommons.org/licenses/by/4.0/

C) The Author(s) 2016 